\title{
Quantifying the global atmospheric power budget
}

\author{
Anastassia M. Makarieva ${ }^{1,2}$, Victor G. Gorshkov ${ }^{1,2}$, Andrei V. Nefiodov ${ }^{1}$, Douglas Sheil ${ }^{3}$, Antonio \\ Donato Nobre ${ }^{4}$, and Bai-Lian $\mathrm{Li}^{2}$ \\ ${ }^{1}$ Theoretical Physics Division, Petersburg Nuclear Physics Institute, 188300 Gatchina, St. Petersburg, Russia \\ ${ }^{2}$ USDA-China MOST Joint Research Center for AgroEcology and Sustainability, University of California, Riverside \\ 92521-0124, US \\ ${ }^{3}$ Norwegian University of Life Sciences, Ås, Norway \\ ${ }^{4}$ Centro de Ciência do Sistema Terrestre INPE, São José dos Campos SP 12227-010, Brazil
}

Correspondence to: Anastassia Makarieva (ammakarieva@gmail.com), Victor Gorshkov (vigorshk@thd.pnpi.spb.ru), Andrei Nefiodov (anef@thd.pnpi.spb.ru), Douglas Sheil (douglas.sheil@nmbu.no), Antonio Donato Nobre (anobre27@gmail.com), Bai-Lian Li (bai-lian.li@ucr.edu)

\begin{abstract}
Starting from the definition of mechanical work for an ideal gas, we present a novel derivation linking global wind power to measurable atmospheric parameters. The resulting expression distinguishes three components: the kinetic power associated with horizontal motion, the kinetic power associated with vertical motion and the gravitational power of precipitation. We discuss the caveats associated with integration of material derivatives in the presence of phase transitions and how these affect published analyses of global atmospheric power. Using the MERRA database for the years 2009-2015 (three hourly data on the $1.25^{\circ} \times 1.25^{\circ}$ grid at 42 pressure levels) we estimate total atmospheric power at $3.1 \mathrm{~W} \mathrm{~m}^{-2}$ and kinetic power at $2.6 \mathrm{~W} \mathrm{~m}^{-2}$. The difference between the two $\left(0.5 \mathrm{~W} \mathrm{~m}^{-2}\right)$ is about half the independently estimated gravitational power of precipitation $\left(1 \mathrm{~W} \mathrm{~m}^{-2}\right)$. We explain how this discrepancy arises from the limited spatial and temporal resolution of the database. Our analysis suggests that the total atmospheric power calculated with a spatial resolution of the order of one kilometer (thus capturing the small moist convective eddies) should be around $5 \mathrm{~W} \mathrm{~m}^{-2}$. We discuss the physical constraints on global atmospheric power and how considering the dynamic effects of water vapor condensation offers new opportunities.
\end{abstract}

\section{Introduction}

How much power does our atmosphere generate and why? These questions have long challenged theorists (Lorenz, 1967) and have gained renewed significance given how our climate is affected by changes in atmospheric circulation (e.g., Bates, 2012; Shepherd, 2014). A particular problem is the mismatch between model predictions and observed trends (e.g., Kociuba and Power, 2015). While models suggest general circulation should slow as global temperatures rise, independent observations indicate circulation is getting faster (e.g., de Boisséson et al., 2014). Global wind power appears to be rising as well (Huang and McElroy, 2015). On the other hand, global circulation models tend to overestimate wind power (Boer and Lambert, 2008). For example, using the CAM3.5 model Marvel et al. (2013) estimated the global kinetic power of the atmosphere ${ }^{1}$ at $3.4 \mathrm{~W} \mathrm{~m}^{-2}$,

\footnotetext{
${ }^{1}$ Note however that Boville and Bretherton (2003) cited $2 \mathrm{~W} \mathrm{~m}^{-2}$ as the kinetic power for the same model. To our knowledge, the inconsistency between their estimate and that of Marvel et al. (2013) has not been addressed.
} 
Atmos. Chem. Phys. Discuss., doi:10.5194/acp-2016-203, 2016

Manuscript under review for journal Atmos. Chem. Phys.

while published observational estimates range from 2 to $2.5 \mathrm{~W} \mathrm{~m}^{-2}$ (Kim and Kim, 2013; Schubert and Mitchell, 2013; Huang and McElroy, 2015).

Our motivation here is to clarify what is meant by total atmospheric power and how we can assess it. Suppose that we know at a given moment in time the air velocity and pressure gradient for each point in the atmosphere with good resolution. Would this information be sufficient to estimate the instantaneous global atmospheric power? We found that for us at least the answer to this question was not self-evident. In Section 2 we explore how the derivation of an expression for the global atmospheric power is affected by the presence of phase transitions.

In Section 3 we show how the derived relationships require a revision of the recent estimates of atmospheric wind power proposed by Laliberté et al. (2015). In Section 4 we discuss how the global wind power in a hydrostatic atmosphere can be represented as a sum of three distinct physical components. Two components dominate in a hydrostatic atmosphere: the kinetic power of the wind generated by horizontal pressure gradients and the gravitational power of precipitation generated by the ascending air. In Section 5 we illustrate our formulations by analysing the atmospheric power budget using the MERRA database (Rienecker et al., 2011). We discuss how estimates of atmospheric power are affected by resolution of the data.

In the concluding section we discuss theoretical constraints on atmospheric power. There are two problems: to explain why wind power on Earth is significantly different from zero (minimal threshold) and to find a constraint from above on this power (maximum threshold). We discuss the opportunities provided by consideration of the dynamic effects of condensation in combination with conventional thermodynamic approach.

\section{Atmospheric power in the presence of phase transitions}

The atmosphere comprises compressible gases and small amounts of incompressible solid and liquid water. We begin with calculating work performed by the atmospheric gases. We treat them as ideal gases with the equation of state

$p=N R T$.

Here $T$ is temperature, $N$ is air molar density $\left(\mathrm{mol} \mathrm{m}^{-3}\right), V \equiv N^{-1}$ is the atmospheric volume occupied by one mole of air, $p$ is air pressure and $R=8.3 \mathrm{~J} \mathrm{~mol}^{-1} \mathrm{~K}^{-1}$ is the universal gas constant.

Work per unit time (power) of an air parcel containing $\tilde{N}$ moles and occupying volume $\tilde{V} \equiv \tilde{N} V\left(\mathrm{~m}^{3}\right)$ is

$p \frac{d \tilde{V}}{d t}=p\left(\tilde{N} \frac{d V}{d t}+V \frac{d \tilde{N}}{d t}\right)=\tilde{V}\left(-\frac{d p}{d t}+R N \frac{d T}{d t}\right)+R T \frac{d \tilde{N}}{d t}$.

We now consider an atmosphere composed of $n$ air parcels with total volume $\mathcal{V} \equiv \sum_{i=1}^{n} \tilde{V}_{i}=\int_{\mathcal{V}} d \mathcal{V}$ (subscript $i$ refers to the $i$-th air parcel). We define $\mathcal{V}$ as the volume bounded by the Earth's surface and the surface corresponding to some fixed pressure level $p_{T}$ at the top of the atmosphere, e.g. to $p_{T}=0.1 \mathrm{hPa}$. This is the uppermost level in many atmospheric datasets including those in the MERRA project.

The number of molecules (moles) $\tilde{N}$ in each air parcel can only change via an inflow (outflow) of molecules through the parcel's boundary. This change results from either diffusion of molecules between the adjacent parcels or from phase 
Atmos. Chem. Phys. Discuss., doi:10.5194/acp-2016-203, 2016

Manuscript under review for journal Atmos. Chem. Phys.

Published: 5 April 2016

(c) Author(s) 2016. CC-BY 3.0 License.
Atmospheric 을

Chemistry

and Physics

Discussions

(c) $\underset{\mathrm{BY}}{(i)}$

transitions or from both. Since in the case of diffusion any molecule leaving one parcel, $d \tilde{N}_{1} / d t<0$, arrives to some other parcel, $d \tilde{N}_{2} / d t=-d \tilde{N}_{1} / d t>0$, all the diffusion terms cancel in the global sum of the last term in Eq. (2) over all parcels. What remains corresponds to phase transitions:

$\sum_{i=1}^{n} \frac{d \tilde{N}_{i}}{d t}=\int_{\mathcal{V}} \frac{1}{\tilde{V}} \frac{d \tilde{N}}{d t} d \mathcal{V}=\int_{\mathcal{V}} \dot{N} d \mathcal{V}$

where $\dot{N}$ is the molar rate of phase transitions per unit volume $\left(\mathrm{mol} \mathrm{m}^{-3} \mathrm{~s}^{-1}\right)$. Its integral over volume $\mathcal{V}$ is equal to the total rate of phase transitions in all the $n$ air parcels. By virtue of the conservation relationship (3) $\dot{N}$ includes the inflow (outflow) into all the air parcels from all liquid or solid surfaces (droplet surface in the atmospheric interior or the Earth's surface).

Using Eqs. (2) and (3) we can write total power $W$ of the $n$ air parcels composing the atmosphere as

$W \equiv \frac{1}{\mathcal{S}} \sum_{i=1}^{n} p_{i} \frac{d \tilde{V}_{i}}{d t}=\frac{1}{\mathcal{S}} \int_{\mathcal{V}} p \frac{1}{\tilde{V}} \frac{d \tilde{V}}{d t} d \mathcal{V}=\frac{1}{\mathcal{S}} \int_{\mathcal{V}}\left(-\frac{d p}{d t}+R N \frac{d T}{d t}+R T \dot{N}\right) d \mathcal{V}$.

Here $\mathcal{S}$ is the Earth's surface area and $W$ is in $\mathrm{W} \mathrm{m}^{-2}$.

The exact differential $d X / d t$ for $X=\{p, T, V, \ldots\}$ that describes change within each air parcel in the atmospheric context corresponds to the material derivative:

$\frac{d X}{d t} \equiv \frac{\partial X}{\partial t}+\mathbf{v} \cdot \nabla X$

where $\mathbf{v}$ is the mean velocity of gas molecules within the parcel.

Using the continuity equation

$\dot{N}=\frac{\partial N}{\partial t}+\nabla \cdot(N \mathbf{v})=\frac{d N}{d t}+N(\nabla \cdot \mathbf{v})$

and the ideal gas law (1) in the form $d p / d t=R N d T / d t+R T d N / d t$ we obtain for $W$ from Eq. (4):

$W=\frac{1}{\mathcal{S}} \int_{\mathcal{V}} p \frac{1}{\tilde{V}} \frac{d \tilde{V}}{d t} d \mathcal{V}=\frac{1}{\mathcal{S}} \int_{\mathcal{V}} p(\nabla \cdot \mathbf{v}) d \mathcal{V}$

The magnitude of $\nabla \cdot \mathbf{v}$ is the rate of relative volume change of a material element at a given point (e.g., Batchelor, 2000, p. 75). This change, $(1 / \tilde{V}) d \tilde{V} / d t$ depends only on the difference in velocities of its bounding material surfaces which are not directly impacted by phase transitions. Therefore, $W$ (7) does not explicitly depend on $\dot{N}$.

We can use the divergence theorem (Gauss-Ostrogradsky theorem) to re-write $W$ (7) as

$$
\begin{aligned}
& W=I_{S}-\frac{1}{\mathcal{S}} \int_{\mathcal{V}}(\mathbf{v} \cdot \nabla p) d \mathcal{V}=I_{S}-\frac{1}{\mathcal{S}} \int_{\mathcal{V}}(\mathbf{u} \cdot \nabla p+\mathbf{w} \cdot \nabla p) d \mathcal{V}, \\
& I_{S} \equiv \frac{1}{\mathcal{S}} \int_{\mathcal{S}} p(\mathbf{v} \cdot \mathbf{n}) d \mathcal{S}=\frac{p_{T}}{\mathcal{S}} \int_{z=z\left(p_{T}\right)}(\mathbf{v} \cdot \mathbf{n}) d \mathcal{S} .
\end{aligned}
$$

Here $\mathbf{n}$ is the outward-pointing unit vector perpendicular to the surface area $d \mathcal{S}$; the surface integral is taken over the Earth's surface and the upper boundary $z=z\left(p_{T}\right)$, where $z\left(p_{T}\right)$ is the altitude of the pressure level $p=p_{T}$. Since the macroscopic 
Atmos. Chem. Phys. Discuss., doi:10.5194/acp-2016-203, 2016

Manuscript under review for journal Atmos. Chem. Phys.

air parcels do not penetrate the Earth's surface ${ }^{2}$, for $z=0$ we have $\mathbf{v} \cdot \mathbf{n}=0$. At the upper boundary $\mathbf{v}$ is zero in the steady state only, when the total volume $\mathcal{V}$ of the air parcels does not change. Generally for $z=z\left(p_{T}\right)$ we have $\mathbf{v} \cdot \mathbf{n} \neq 0$ (a similar condition of non-zero velocity at the upper boundary (the oceanic surface) is commonly used in oceanic science, e.g. Tailleux (2015)).

\section{Practical implications of the obtained relationships}

\subsection{The material derivative of enthalpy}

In a recent effort to constrain the atmospheric power budget, Laliberté et al. (2015) used the thermodynamic identity

$T \frac{d s}{d t} \equiv \frac{d h}{d t}-\frac{1}{\rho} \frac{d p}{d t}+\mu \frac{d q_{T}}{d t}$,

where $s$ is entropy, $h$ is enthalpy, $\mu$ is chemical potential (all per unit mass of wet air), $1 / \rho$ is specific air volume and $q_{T}$ is the mass fraction of total water ${ }^{3}$. Laliberté et al. (2015) neglected, as we do, the atmospheric liquid and solid water content ${ }^{4}$ and approximated $q_{T}=q_{v}$, where $q_{v}$ is the mass fraction of water vapor.

When integrating Eq. (10) over atmospheric mass, Laliberté et al. (2015) assumed that the enthalpy term vanishes, $\int_{\mathcal{M}}(d h / d t) d \mathcal{M}=$ 0 . This assumption was justified by noting that the atmosphere is approximately in a steady state. However, the correct condition for the steady atmospheric state is different. It corresponds to zero enthalpy change in all air parcels combined:

$$
\frac{\partial h_{t o t}}{\partial t}=\sum_{i=1}^{n} \frac{d \tilde{h}_{i}}{d t}=0, h_{t o t} \equiv \sum_{i=1}^{n} \tilde{h}_{i}, \tilde{h}_{i} \equiv m_{i} h_{i} .
$$

where $d \tilde{h}_{i} / d t$ is the change of enthalpy $\tilde{h}_{i}$ of the $i$-th air parcel which has mass $m_{i}$ and mass-specific enthalpy $h_{i}$.

Repeating the reasoning from the previous section we have

$$
\frac{\partial h_{t o t}}{\partial t}=\sum_{i=1}^{n} \frac{d \tilde{h}_{i}}{d t}=\sum_{i=1}^{n}\left(h_{i} \frac{d m_{i}}{d t}+m_{i} \frac{d h_{i}}{d t}\right)=\int_{\mathcal{V}} h \dot{\rho} d \mathcal{V}+\int_{\mathcal{M}} \frac{d h}{d t} d \mathcal{M}
$$

where $\dot{\rho}$ is the mass rate of phase transitions per unit volume $\left(\mathrm{kg} \mathrm{m}^{-3} \mathrm{~s}^{-1}\right)$ obeying the steady-state continuity equation

$\nabla \cdot(\rho \mathbf{v})=\dot{\rho}$.

\footnotetext{
${ }^{2}$ The molecular process of evaporation from the Earth's surface can be viewed as an upward flux of gaseous molecules that are concentrated within one free path length $l$ from the evaporating surface and have mean vertical velocity $w_{E}$ of the order of mean molecular velocity. Molar density $N_{E}$ of such molecules is obtained from evaporation rate $E\left(\mathrm{~kg} \mathrm{~m}^{-2} \mathrm{~s}^{-1}\right)$ as $N_{E} w_{E}=E / M_{v}$, where $M_{v}$ is molar mass of water. Due to the presence of these molecules, macroscopic air parcels adjacent to the surface could have a non-zero vertical velocity $w_{s}$ defined as the mean vertical velocity of all molecules in the parcel. If $l_{p}$ is the linear size of this parcel, we have $w_{s}=w_{E} N_{E} l /\left(N_{s} l_{p}\right)$, where $N_{s}=p_{s} /\left(R T_{s}\right)$ is molar density of air at the surface, $T_{s}$ and $p_{s}$ are surface temperature and pressure. Then for the vertical term $p(\mathbf{v} \cdot \mathbf{n})$ at the surface, Eq. (9), we have $p_{s} w_{s}=\left(l / l_{p}\right) E R T_{s} / M_{v}$. Since as we will see in Sections 5 and 6 global atmospheric power is of the order of $P R T_{s} / M_{v}$, where $P=E$ is the global mean precipitation and evaporation, the surface term $p_{s} w_{s}$ can be neglected in Eq. (9) if $l / l_{p} \ll 1$, i.e. on any macroscopic length scale.

${ }^{3}$ The unconventional sign at the chemical potential term follows from $\mu$ being defined in Eq. (10) relative to dry air: hence, when the relative dry air content diminishes this term is negative. For details see p. 8 in the Supplementary Materials of Laliberté et al. (2015).

${ }^{4}$ This assumption corresponds to an instantaneous removal of the non-gaseous water from the atmosphere by precipitation.
} 
Atmos. Chem. Phys. Discuss., doi:10.5194/acp-2016-203, 2016

Manuscript under review for journal Atmos. Chem. Phys.

As is clear from Eq. (11), when the atmosphere is in a steady state $\left(\partial h_{t o t} / \partial t=0\right)$ the mass integral of the material derivative of enthalpy is not zero. It can be roughly estimated by assuming that all evaporation occurs at the surface $z=0$, while all condensation occurs at mean condensation height $z=\mathcal{H}_{P}$ :

$\dot{\rho}(x, y, z)=E(x, y) \delta(z)-P(x, y) \delta\left(z-\mathcal{H}_{P}\right), E=P, E \equiv \frac{1}{\mathcal{S}} \int_{\mathcal{S}} E(x, y) d \mathcal{S}, P \equiv \frac{1}{\mathcal{S}} \int_{\mathcal{S}} P(x, y) d \mathcal{S}$,
$\frac{1}{\mathcal{S}} \int_{\mathcal{M}} \frac{d h}{d t} d \mathcal{M}=-\frac{1}{\mathcal{S}} \int_{\mathcal{V}} h \dot{\rho} d \mathcal{V} \approx-E h_{s}+P h\left(\mathcal{H}_{P}\right) \equiv-P \Delta h_{c}, \Delta h_{c} \equiv h_{s}-h\left(\mathcal{H}_{P}\right), h=c_{p} T+L q_{v}$.

Here $E(x, y)$ and $P(x, y)$ are local evaporation and precipitation at the surface $\left(\mathrm{kg} \mathrm{m}^{-2} \mathrm{~s}^{-1}\right)$ with global averages $E$ and $P$; subscript $s$ denotes surface values $z=0 ; \delta(z)$ is the Dirac delta function; $c_{p}=10^{3} \mathrm{~J} \mathrm{~kg}^{-1} \mathrm{~K}^{-1}$ is heat capacity of air at constant pressure, $L=2.5 \times 10^{6} \mathrm{~J} \mathrm{~kg}^{-1}$ is latent heat of vaporization. For $\mathcal{H}_{P} \approx 2.5 \mathrm{~km}$ (Makarieva et al., 2013a) and $q_{v}\left(\mathcal{H}_{P}\right) \ll q_{v s}$ we have $-P \Delta h_{c}=-P\left(c_{p} \mathcal{H}_{P} \Gamma+L q_{v s}\right) \approx-1 \mathrm{~W} \mathrm{~m}^{-2}$. Here $q_{v s}=0.0083$ corresponds to global mean surface temperature $T_{s}=$ $288 \mathrm{~K}$ and relative humidity $80 \%$; mean tropospheric lapse rate is $\Gamma=6.5 \mathrm{~K} \mathrm{~km}^{-1}$. Global mean precipitation $P$ (measured in a system of units where liquid water density $\rho_{w}=10^{3} \mathrm{~kg} \mathrm{~m}^{-3}$ is set to unity) is equal to $P \sim 1 \mathrm{~m}$ year ${ }^{-1}$, which in SI units corresponds to $P=3.2 \times 10^{-5} \mathrm{~kg} \mathrm{~m}^{-2} \mathrm{~s}^{-1}$. A more sophisticated estimate ${ }^{5}$ of integral (15) presented in Appendix A yields $-1.6 \mathrm{~W} \mathrm{~m}^{-2}$ with an accuracy of about $30 \%$.

These estimates show that the enthalpy term cannot be neglected in Eq. (10) on either theoretical or quantitative grounds. By absolute magnitude the integral (15) is greater than one third of the total atmospheric power $W \approx 4 \mathrm{~W} \mathrm{~m}^{-2}$ estimated by Laliberté et al. (2015) for the MERRA re-analysis $\left(3.66 \mathrm{~W} \mathrm{~m}^{-2}\right)$ and the CESM model $\left(4.01 \mathrm{~W} \mathrm{~m}^{-2}\right)$.

Laliberté et al. (2015) first calculated the mass integral of $T d s / d t$ from the right-hand side of Eq. (10), then calculated $\mu d q_{T} / d t$ from atmospheric data and then used the obtained values and again Eq. 10 to estimate the total atmospheric power as $-(1 / \mathcal{S}) \int_{\mathcal{M}}(1 / \rho)(d p / d t) d \mathcal{M}$. In such a procedure, putting $\int_{\mathcal{M}}(d h / d t) d \mathcal{M}=0$ should have overestimated $W$ by about $1.6 \mathrm{~W} \mathrm{~m}^{-2}$. Since the omitted term is proportional to the global precipitation rate, it is crucial not only for a correct estimate of the mean value of $W$, but also for the determination of any trends related to precipitation.

Note also that even in the correct form, with the enthalpy term retained, Eq. (10) does not provide a theoretical constraint on $W$. This equation is an identity: it essentially defines $d s / d t$ in terms of measurable atmospheric data. As is clear from Eq. (8), $W$ can be estimated from the same data directly without involving entropy, see Section 5.

\subsection{Material derivative of pressure $d p / d t$ and atmospheric power}

Equations (2)-(8) clarify the relationship between atmospheric power and $d p / d t\left(\mathrm{~W} \mathrm{~m}^{-3}\right)$. Global atmospheric power $W$ is, in the general case, not equal to the mass integral of $-(1 / \rho) d p / d t$ (or volume integral of $d p / d t$, which is the same since

\footnotetext{
${ }^{5}$ The physical meaning of the negative sign of the enthalpy integral (15) can be understood as follows. This integral describes how much air belongs to the air parcels decreasing their enthalpy $(d h / d t<0)$ compared to how much air belongs to the air parcels increasing their enthalpy $(d h / d t>0)$. In the absence of phase transitions these masses are equal. There is as much gas going upwards, as there is going downwards. Hence, $\int_{\mathcal{M}}(d h / d t) d \mathcal{M}=0$. In the presence of condensation and evaporation, these masses are not equal. Water vapor is "created" by evaporation at the planetary surface and then "destroyed" by condensation at some height $\mathcal{H}_{P}>0$. In the result, at any height $z>0$ there is, on average, more gas going upwards than downwards. In the ascending air $d h / d t<0$ (it cools and loses water vapor). Therefore, $\int_{\mathcal{M}}(d h / d t) d \mathcal{M}<0$.
} 
Atmos. Chem. Phys. Discuss., doi:10.5194/acp-2016-203, 2016

Manuscript under review for journal Atmos. Chem. Phys.

$d \mathcal{M}=\rho d \mathcal{V}$ ). While the definition of material derivative (5) includes partial derivative over time, we have seen that atmospheric work output $W$ does not depend on $\partial p / \partial t$, see Eqs. (8), (7). Indeed, from Eq. (8) and (5) we find:

$W=-\frac{1}{\mathcal{S}} \int_{\mathcal{V}} \frac{d p}{d t} d \mathcal{V}+\frac{1}{\mathcal{S}} \int_{\mathcal{V}} \frac{\partial p}{\partial t} d \mathcal{V}+I_{S}$.

In the steady state, when the volume of the atmosphere is constant, the last two terms in the right-hand side of Eq. (16) are zero. Since the distribution of pressure versus altitude in the atmosphere is approximately exponential and since $I_{S}$ is proportional to $p_{T}$, by choosing a sufficiently small upper boundary pressure $p_{T}$ it is possible to ensure that the instantaneous value of $I_{S}$ (9) is arbitrarily small compared to the instantaneous $W$ (see Fig. 6d in Appendix C). Everywhere below we put $I_{S}=0$.

The long-term average of the second integral in the right-hand side of (16) is also zero. However, its instantaneous value depends on temperature tendency $\partial T / \partial t$ and is not necessarily negligible compared to $W$. This term describes pressure changes that are not related to power output but reflect how internal energy of the atmosphere varies with changing atmospheric volume and/or mass (see Appendix C for details).

Locally, even in the stationary case when $\partial p / \partial t=0$, material derivative of pressure $-d p / d t \equiv-\mathbf{v} \cdot \nabla p$ is not equal to power output per unit volume of the local air parcel $(p d \tilde{V} / d t) / \tilde{V}\left(\mathrm{~W} \mathrm{~m}^{-3}\right)$. From Eq. (7), which holds true for an arbitrary volume $\mathcal{V}$, we have

$p \frac{1}{\tilde{V}} \frac{d \tilde{V}}{d t}=p(\nabla \cdot \mathbf{v})=-\mathbf{v} \cdot \nabla p+\nabla \cdot(p \mathbf{v})$.

These magnitudes have different physical meaning. While $p(\nabla \cdot \mathbf{v})$ describes power output per unit volume of the local air parcel, $-\mathbf{v} \cdot \nabla p$, describes power output per unit volume of the local pressure gradient. It is work performed per unit time by the force of the local pressure gradient on the air contained in the considered unit volume. Global integrals of $-\mathbf{v} \cdot \nabla p$ and $p(\nabla \cdot \mathbf{v})$ coincide and equal global atmospheric power $W$ when $I_{\mathcal{S}}$ in $(8)$ is zero.

\section{Revisiting the current understanding of the atmospheric power budget}

We now show how total atmospheric power (8) can be decomposed into distinct terms. In Eq. (8) the expression $-\mathbf{v} \cdot \nabla p=$ $-\mathbf{u} \cdot \nabla p-\mathbf{w} \cdot \nabla p$ represents total work performed by the pressure gradient per unit time per unit air volume. The horizontal pressure gradient generates the kinetic energy of the horizontal wind $\mathbf{u}$. The vertical pressure gradient generates the kinetic energy of the vertical wind $w$ plus it changes the potential energy of air in the gravitational field.

In hydrostatic equilibrium we have

$\nabla_{z} p=\rho \mathbf{g}$

where $\rho=N M$ is air density $\left(\mathrm{kg} \mathrm{m}^{-3}\right), M$ is air molar mass $\left(\mathrm{kg} \mathrm{mol}^{-1}\right)$. In the real atmosphere due to the presence of non-gaseous water the air distribution deviates from Eq. (18) such that we have $\nabla_{z} p=\left(\rho+\rho_{l}\right) \mathbf{g}$ and in Eq. (8) $-\mathbf{w} \cdot \nabla p=$ $-\rho \mathbf{w} \cdot \mathbf{g}-\rho_{l} \mathbf{w} \cdot \mathbf{g}$, where $\rho_{l}$ is mass density of the non-gaseous water in the air. 
Atmos. Chem. Phys. Discuss., doi:10.5194/acp-2016-203, 2016

Term $-\rho \mathbf{w} \cdot \mathbf{g}=-\rho \mathbf{v} \cdot \mathbf{g}$ represents the vertical flux of air: it is positive (negative) for the ascending (descending) air. Recalling that $\mathrm{g}=-g \nabla z$ and using the divergence theorem and the stationary continuity equation (13) we can write

$W_{P} \equiv-\frac{1}{\mathcal{S}} \int_{\mathcal{V}} \rho \mathbf{w} \cdot \mathbf{g} d \mathcal{V}=\frac{1}{\mathcal{S}} g \int_{\mathcal{V}} \rho \mathbf{v} \cdot \nabla z d \mathcal{V}=\frac{1}{\mathcal{S}} g \int_{\mathcal{S}} \mathbf{n} \cdot(\mathbf{v} \rho z) d \mathcal{S}-\frac{1}{\mathcal{S}} g \int_{\mathcal{V}} z \dot{\rho} d \mathcal{V}$.

The surface integral in (19) is taken at the Earth's surface (here it is zero because $z=0$ ) and $z=z\left(p_{T}\right)$ (here it is also zero, because $\rho \mathbf{n} \cdot \mathbf{v}=0$ ).

For a dry atmosphere where $\dot{\rho}=0$, the last volume integral in Eq. (19) is zero and $W_{P}=0$ : indeed, in this case at any height $z$ there is as much air going upwards as there is going downwards ${ }^{6}$. In a moist atmosphere, evaporation $\dot{\rho}>0$ (condensation $\dot{\rho}<0$ ) makes a negative (positive) contribution to $W_{P}$. This is because $W_{P}$ reflects the work of water vapor as it travels from the level where evaporation occurs (where water vapor arises) to the level where condensation occurs (where water vapor disappears). When condensation takes place above where evaporation occurs, the water vapor expands as it moves upwards towards condensation, and the work is positive.

When evaporation occurs at the Earth's surface $z=0, W_{P}(19)$ is equal to $P g \mathcal{H}_{P}$, where $\mathcal{H}_{P}$ is the global mean height of condensation. It is natural to call $W_{P}$ the "gravitational power of precipitation". Pauluis et al. (2000) estimated the value of $W_{P}$ for the tropics. Their estimate was revised by Pauluis and Dias (2012) and by Makarieva et al. (2013a) who also estimated global $W_{P}$ as $0.8 \mathrm{~W} \mathrm{~m}^{-2}$. (Here we revised this latter estimate upwards by $20 \%$, see Appendix A).

The stationary power budget for a hydrostatic atmosphere can be written as

$$
\begin{aligned}
W & =-\frac{1}{\mathcal{S}} \int_{\mathcal{V}} \mathbf{v} \cdot \nabla p d \mathcal{V} \equiv W_{K}+W_{P}, \\
W_{K} & \equiv-\frac{1}{\mathcal{S}} \int_{\mathcal{V}}\left(\mathbf{u} \cdot \nabla p+\rho_{l} \mathbf{w} \cdot \mathbf{g}\right) d \mathcal{V} \approx-\frac{1}{\mathcal{S}} \int_{\mathcal{V}} \mathbf{u} \cdot \nabla p d \mathcal{V}, \\
W_{P} & \equiv-\frac{1}{\mathcal{S}} \int_{\mathcal{V}} \rho \mathbf{w} \cdot \mathbf{g} d \mathcal{V}=-\frac{1}{\mathcal{S}} \int_{\mathcal{V}} g z \dot{\rho} d \mathcal{V}=P g \mathcal{H}_{P}, P \equiv-\frac{1}{\mathcal{S}} \int_{z>0} \dot{\rho} d \mathcal{V} .
\end{aligned}
$$

Equations Eqs. (20)-(22) and their derivation have not been previously published. These equations clarify the physical meaning of the atmospheric power budget. While we define $W(4)$ as the power output of ideal gas parcels, this power includes $W_{P}$ - the gravitational power of precipitation. The meaning is that hydrometeors perform work at the expense of their potential energy. To acquire this energy, a corresponding amount of water vapor must be raised by air parcels. We can also see that $W_{P}$ does not depend on the interaction between the air and the falling hydrometeors. This term would be present in the atmospheric power budget even if hydrometeors were experiencing free fall and did not interact with the air at all (such that no frictional dissipation on hydrometeors occurred).

The term $-\rho_{l} \mathbf{w} \cdot \mathbf{g}$ in Eq. (21) is not related to the gravitational power of precipitation. It describes kinetic energy generation on the vertical scale of the order of the atmospheric scale height $\mathcal{H} \equiv-p /(\partial p / \partial z)=R T /(M g) \sim 10 \mathrm{~km}$. This energy is generated because the vertical air distribution deviates from the hydrostatic equilibrium (18). Hydrometeors act as

\footnotetext{
${ }^{6}$ Note that using the continuity equations for dry air $\nabla \cdot\left(\rho_{d} \mathbf{v}\right)=0$ and water vapor $\nabla \cdot\left(\rho_{v} \mathbf{v}\right)=\dot{\rho}$, where $\rho_{d}$ and $\rho_{v}$ are densities of dry air and water vapor, we find from Eq. (19) that $W_{P}=-(1 / \mathcal{S}) \int_{\mathcal{V}} \rho_{v} \mathbf{w} \cdot \mathbf{g} d \mathcal{V}$.
} 
Atmos. Chem. Phys. Discuss., doi:10.5194/acp-2016-203, 2016

Manuscript under review for journal Atmos. Chem. Phys.

resistance preventing the pressure difference $\Delta p \sim \rho_{l} g \mathcal{H}$ from converting to the kinetic energy of a vertical wind. In the atmosphere on average $\rho_{l} / \rho \sim 10^{-5}$ (Makarieva et al., 2013a). Without hydrometeors, the non-equilibrium pressure difference $\Delta p \sim 10^{-5} \rho g \mathcal{H} \sim 1$ Pa would produce maximum vertical velocity of about $w_{m} \sim 1 \mathrm{~m} \mathrm{~s}^{-1}\left(\rho w_{m}^{2} / 2=\Delta p\right)$. This is two orders of magnitude larger than the characteristic vertical velocities $w \sim 10^{-2} \mathrm{~m} \mathrm{~s}^{-1}$ of large-scale air motions. (Hydrometeors thus inhibit vertical motion in a similar way turbulent friction at the surface inhibit horizontal air motion. For example, the observed meridional surface pressure differences of the order of $\Delta p_{h} \sim 10 \mathrm{hPa}$ in the tropics, if friction were absent, could have produced maximum horizontal air velocities of about $u_{m} \sim 40 \mathrm{~m} \mathrm{~s}^{-1}\left(\rho u_{m}^{2} / 2=\Delta p_{h}\right)$.) Quantitatively, $-\rho_{l} \mathbf{w} \cdot \mathbf{g}$ is less than $1 \%$ of $W$ and can be neglected: its volume integral taken per unit surface area is less than $\rho_{l} g \mathcal{H} w \sim 10^{-5} p w \sim 10^{-2} \mathrm{~W} \mathrm{~m}^{-2}$, where $p=\rho g \mathcal{H}=10^{5} \mathrm{~Pa}$ is air pressure at the surface.

In some publications kinetic power is assumed equal to the volume integral of $d p / d t$ (e.g., Robertson et al., 2011, their Eq. 1). Here we have seen that the long-term mean of this integral is equal to total power $W$, Eq. (16). However, the instantaneous value of this integral is not equal to the instantantaneous value of $W$, see also Appendix (C). In other studies kinetic power is correctly defined and estimated from horizontal velocities as $W_{K}$ (21) (see, e.g., Boville and Bretherton, 2003; Huang and McElroy, 2015). In such studies $W_{K}$ is sometimes confused with total atmospheric power: i.e. in the total power budget the gravitational power of precipitation, $W_{P}$, is overlooked (e.g., Huang and McElroy, 2015, their Fig. 10). We also note that the gravitational power of precipitation $W_{P}$ has not been explicitly identified in past studies assessing the conversion rates between available potential and kinetic energies in the framework of the Lorenz energy cycle (see, e.g., Kim and Kim, 2013, and references therein).

The fact that kinetic power $W_{K}(21)$ depends on horizontal and not vertical velocities is essential for comparing theory and observations. Horizontal pressure gradients and wind velocities are observed directly, while vertical velocities are inferred only indirectly and with significant uncertainty (see Appendix C).

\section{Observation-based estimates of global atmospheric power}

\subsection{Total power $W$}

In meteorological databases including the MERRA dataset MAI3CPASM that we used (see Appendix B for details), $d p / d t$ is often represented as a separate variable named pressure velocity (omega). We estimated $W$ as

$W=\langle\Omega\rangle, \Omega \equiv-\frac{1}{\mathcal{S}} \int_{\mathcal{V}} \omega d \mathcal{V}, \omega \equiv \frac{d p}{d t} \equiv \frac{\partial p}{\partial t}+\mathbf{v} \cdot \nabla p$.

Time averaging denoted as \langle\rangle was made over seven years, 2009-2015. Using instantaneous omega values provided each three hours at 42 pressure levels on the $1.25^{\circ} \times 1.25^{\circ}$ grid we obtained two estimates, $W_{1}=2.97 \mathrm{~W} \mathrm{~m}^{-2}$ and $W_{2}=3.24 \mathrm{~W} \mathrm{~m}^{-2}$ depending on how the local omega value at the Earth's surface was estimated. The value of $W_{1} \equiv\left\langle\Omega_{1}\right\rangle$ was obtained by linearly extrapolating the omega value from the two nearest pressure levels, see Eq. (B3), while $W_{2} \equiv\left\langle\Omega_{2}\right\rangle$ was obtained assuming 
Atmos. Chem. Phys. Discuss., doi:10.5194/acp-2016-203, 2016

Manuscript under review for journal Atmos. Chem. Phys.

Table 1. MERRA-based and theory-derived estimates of the global atmospheric power budget $W=W_{K}+W_{P}\left(\mathrm{~W} \mathrm{~m}^{-2}\right)$ and the enthalpy term $-P \Delta h_{c}(15)\left(\mathrm{W} \mathrm{m}^{-2}\right)$. Subscripts 1 and 2 refer to the two means of estimating $W$ and $W_{K}$, see Section 5 and Appendix B, Eqs. (B3), (B12) for 1 and (B4), (B13) for 2 .

\begin{tabular}{llllll}
\hline$\#$ & Source of estimate & $W$ & $W_{K}$ & $W_{P}$ & $-P \Delta h_{c}$ \\
\hline 1 & MERRA 2009-2015 $\left(W_{1}, W_{K 1}\right)$ & 2.97 & 2.70 & $0.27^{*}$ & $-0.69^{\dagger}$ \\
2 & MERRA 2009-2015 $\left(W_{2}, W_{K 2}\right)$ & 3.24 & 2.53 & $0.71^{*}$ & $-0.42^{\dagger}$ \\
3 & MERRA 2009-2015 (mean) & 3.1 & 2.6 & $0.5^{*}$ & $-0.6^{\dagger}$ \\
4 & Theoretical estimates based on $P=0.96$ m year $^{-1}$ & $4.8^{\text {ब }}$ & $3.8^{\S}$ & $1.0^{\ddagger}$ & $-1.6^{\ddagger}$ \\
5 & Relative difference $(\# 4-\# 3) / \# 3$ & 0.4 & 0.3 & 0.5 & 0.6 \\
\hline
\end{tabular}

* estimated as the difference between MERRA-based $W$ and $W_{K} ;{ }^{\dagger}$ estimated as the difference between MERRA-based $W$ and

$W_{L}=3.66 \mathrm{~W} \mathrm{~m}^{-2}$ of Laliberté et al. (2015); ${ }^{\ddagger}$ estimated in Appendix A; ${ }^{\S}$ estimated from Eq. (25); ${ }^{\Uparrow}$ estimated as the sum of the theoretical $W_{K}$ and $W_{P}$.

that omega at the surface is equal to the surface pressure tendency $\partial p_{s} / \partial t$, see Eq. (B4). The results are shown in Fig. 1a and Table 1.

Laliberté et al. (2015) using their Eq. (10) estimated total atmospheric power from the MERRA database as $W_{L}=3.66 \mathrm{~W} \mathrm{~m}^{-2}$. Both $W_{1}$ and $W_{2}$ are significantly smaller. We have $W_{1}-W_{L}=-0.69 \mathrm{~W} \mathrm{~m}^{-2}(19 \%)$ and $W_{2}-W_{L}=-0.42 \mathrm{~W} \mathrm{~m}^{-2}(12 \%)$. As discussed in Section 3, the difference between $W$ and $W_{L}$, caused by the omission of the enthalpy term in Eq. (10), should be equal to the enthalpy term (15) of about $-1.6 \mathrm{~W} \mathrm{~m}^{-2}$. The actual difference is the same order of magnitude with our estimate (15) but is about $60 \%$ smaller, Table 1.

This discrepancy may be a consequence of Laliberté et al. (2015) using different MERRA datasets (MAI3NECHM, MAI6NVANA, MAT3NVCHM). These data have a $0.67^{\circ} \times 0.5^{\circ}$ lon-lat resolution and are provided on 72 vertical levels. However, pressure velocity is not provided. It was calculated by Laliberté et al. (2015) from horizontal velocities using a correction procedure to ensure mass conservation (see the Supplementary Materials of Laliberté et al., 2015, for details). The impact of this procedure on the obtained results was not investigated. We employed the "ready-to-use" omega values from the MERRA dataset providers. Another reason for the discrepancy is the resolution of the data - this is a topic we examine further below.

\subsection{Kinetic power $W_{K}$}

Two estimates of kinetic power were obtained (Fig. 1a and Table 1): $W_{K 1}=2.70 \mathrm{~W} \mathrm{~m}^{-2}$ by extrapolating $\mathbf{u} \cdot \nabla p$ linearly to the surface from the two nearest pressure levels, see Eq. (B12), and $W_{K 2}=2.53 \mathrm{~W} \mathrm{~m}^{-2}$ assuming $\mathbf{v}=0$ and $\mathbf{u} \cdot \nabla p=0$ at the surface, see Eq. (B13). The second estimate is close to the long-term mean kinetic power $2.46 \mathrm{~W} \mathrm{~m}^{-2}$ obtained by Huang and McElroy (2015) for 1979-2010.

The difference between the estimates $W_{1}$ and $W_{2}$ and $W_{K 1}$ and $W_{K 2}$ stems from different assumptions about the value of the integrated quantity ( $\omega$ or $\mathbf{u} \cdot \nabla p$ ) at the surface. The surface layer averages about $13 \mathrm{hPa}$ higher pressure than $p_{1}=1000 \mathrm{hPa}$, 
Atmos. Chem. Phys. Discuss., doi:10.5194/acp-2016-203, 2016

Manuscript under review for journal Atmos. Chem. Phys.

Published: 5 April 2016

(c) Author(s) 2016. CC-BY 3.0 License.
Atmospheric

Chemistry

and Physics

Discussions

(c) $\underset{\mathrm{BY}}{(i)}$
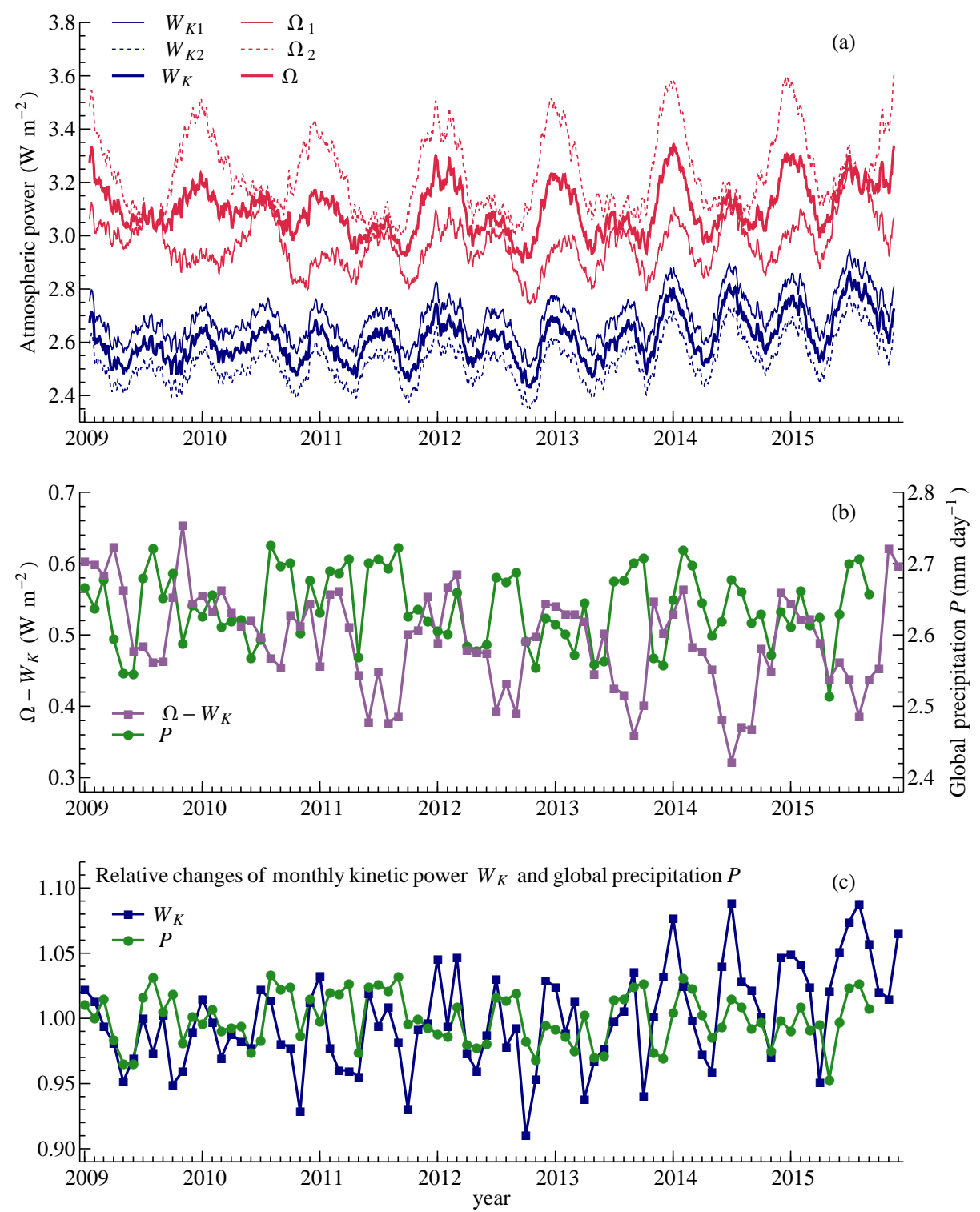

Figure 1. (a) Time series (60-day running mean of daily values) of the kinetic power (the blue (lower) curves) and the omega integral (23), (B6) corresponding to total atmospheric power (the red (upper) curves) (thin solid curves: $\Omega_{1}, W_{K 1}$; dashed curves $\Omega_{2}, W_{K 2}$; thick solid curves $\Omega \equiv\left(\Omega_{1}+\Omega_{2}\right) / 2, W_{K} \equiv\left(W_{K 1}+W_{K 2}\right) / 2$ ). (b) Monthly mean values of $\Omega-W_{K}$ and global precipitation $P$ (Adler et al., 2003, GPCP v. 2.2). (c) Relative changes of kinetic power $W_{K}$ and global precipitation $P$ (monthly $W_{K}$ and $P$ values divided by their long-term mean of, respectively, $2.6 \mathrm{~W} \mathrm{~m}^{-2}$ for $W_{K}$ in 2009-2015 and $2.64 \mathrm{~mm} \mathrm{day}^{-1}$ for $P$ in 2009-2014). Ticks on the horizontal axes correspond to the 15 th day of each month. 
Atmos. Chem. Phys. Discuss., doi:10.5194/acp-2016-203, 2016

Manuscript under review for journal Atmos. Chem. Phys.

the pressure of the first layer in the MERRA database. This difference corresponds to an atmospheric layer about $H_{1} \sim 100 \mathrm{~m}$ thick. Estimates $W_{2}$ and $W_{K 2}$ are based on the assumption that at the surface $\mathbf{v}=0$ and $\mathbf{u} \cdot \nabla p=0$ (see Appendix B). However, within the boundary layer air velocity becomes essentially non-zero reaching a few meters per second at a height $H_{v}$ of the order of a few meters (e.g., Beare et al., 2006). Thus, linear extrapolation from $\mathbf{v}=0$ at the surface to its known value at pressure level $p_{1}$, i.e. from $z=0$ to $z=H_{1} \gg H_{v}$, does not accurately reflect the velocity profile of the surface layer between $p_{s}$ and $p_{1}$. Estimates $W_{1}$ and $W_{K 1}$, on the other hand, assume that within the surface layer the integrated quantity varies in the vertical in the same manner as it does between the two pressure levels nearest to the surface (e.g., between $p_{1}=1000 \mathrm{hPa}$ and $p_{2}=975 \mathrm{hPa}$ ), see Eqs. (B3) and (B12). With increasing vertical resolution, the two estimates should coincide. At present resolution, they differ by $10 \%$ for $W_{1}$ and $W_{2}$ and by $6 \%$ for $W_{K 1}$ and $W_{K 2}$. Lacking better guidance, we will use the mean of the two estimates as a representative value. We then have $W=\left(W_{1}+W_{2}\right) / 2=3.1 \mathrm{~W} \mathrm{~m}^{-2}$ and $W_{K}=\left(W_{K 1}+W_{K 2}\right) / 2=2.6 \mathrm{~W} \mathrm{~m}^{-2}$, Table 1.

The interannual variation of global atmospheric power is relatively small. Total power $W$ had its minimum of $3.05 \mathrm{~W} \mathrm{~m}^{-2}$ in 2011 and maximum of $3.21 \mathrm{~W} \mathrm{~m}^{-2}$ in 2015. Kinetic power $W_{K}$ had its minimum of $2.57 \mathrm{~W} \mathrm{~m}^{-2}$ in 2009 and 2011 and maximum of $2.72 \mathrm{~W} \mathrm{~m}^{-2}$ in 2015 , see Table 3 in Appendix B.

While the global mean values of $W$ and $W_{K}$ differ by a relatively small margin, their local values differ by more than an order of magnitude and have different spatial distributions, Fig. 2. Total atmospheric power is positive (negative) in the regions of ascent (descent); local values may exceed the global mean by nearly two orders of magnitude. The kinetic power is more spatially uniform. It is nearly ubiquitously positive in the lower atmosphere below the $800 \mathrm{hPa}$ pressure level: $58 \%$ of global kinetic power is generated below $800 \mathrm{hPa}$. In the upper atmosphere negative kinetic power occurs in the region of the atmospheric heat pumps (Ferrel cells) (Makarieva et al., 2015c).

The difference between $W$ and $W_{K}$ should be equal to the gravitational power of precipitation $W_{P}$, Eq. (20). As estimated from the MERRA database, this difference amounts to $0.5 \mathrm{~W} \mathrm{~m}^{-2}$, Table 1 . This is half the independent estimate of $W_{P}=$ $1 \mathrm{~W} \mathrm{~m}^{-2}$ obtained using the observed precipitation, see Appendix A.

The shortfall in our estimate arises from the coarse resolution in the MERRA database. In convection eddies, air rises approximately moist adiabatically and descends dry adiabatically. Within such eddies, provided most condensed moisture is removed by precipitation, the water vapor mixing ratio decreases in the ascending air and remains approximately constant in the descending air. In consequence, if we average over many such eddies we will find that the mean mixing ratio decreases with height. Now, if the considered area has on average a negative large-scale vertical velocity $w<0$, this means that in this descending air the mixing ratio is increasing - this is equivalent to an atmospheric source of water vapor. Here we will call this artifact "spurious evaporation". Since according to Eq. (22) evaporation within the atmosphere reduces $W_{P}$, averaging over an area larger than that of individual convective eddies $(\sim 1-10 \mathrm{~km})$ underestimates $W_{P}$.

The difference between the monthly mean values of the volume integral of pressure velocity (23) and $W_{K}$ does not correlate positively with monthly mean precipitation (as $W_{P}$ should). In particular, the summer maximum of precipitation corresponds to a minimum of $\Omega-W_{K}$, Fig. 1b. One possible reason is that the integral of the pressure tendency $\partial p / \partial t$ that distinguishes $\Omega$ (23) from the instantaneous value of total atmospheric power (16), does not vanish on the seasonal timescale thus significantly 
Atmos. Chem. Phys. Discuss., doi:10.5194/acp-2016-203, 2016

Manuscript under review for journal Atmos. Chem. Phys.

Published: 5 April 2016

(c) Author(s) 2016. CC-BY 3.0 License.
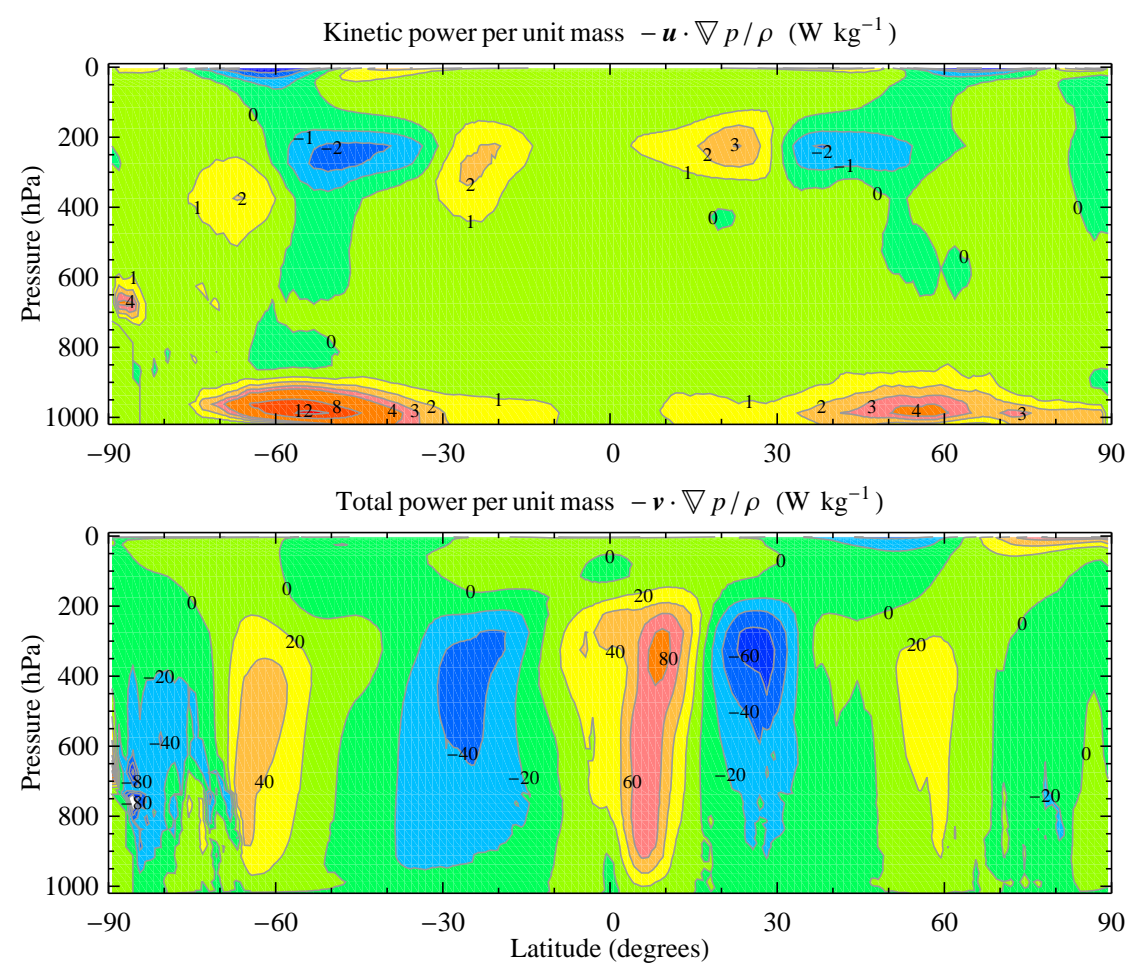

Figure 2. The zonally averaged vertical profile of kinetic $W_{K}$ and total $W$ atmospheric power versus latitude. Mean values for 2009-2015 are shown.

affecting the monthly estimate of atmospheric power. In Appendix $\mathrm{C}$ we consider the seasonal cycle of the volume integral of the pressure tendency and evaluate its magnitude in greater detail.

Kinetic power $W_{K}$, on the other hand, is better correlated with global precipitation, Fig. 1c and Fig. 3. Relative changes of $W_{K}$ are the same order of magnitude as changes of $P$. Both $W_{K}$ and $P$ have two peaks, one in summer and another in winter, Fig. 3.

\section{Discussion and conclusions: Towards constraining the atmospheric power}

We have shown how atmospheric power $W$ can be defined in terms of measurable atmospheric variables, Eqs. (20)-(22). We used the MERRA database to quantify the atmospheric power budget, Table 1 . We found that the gravitational power of precipitation $W_{P}$ estimated as the difference between total power $W$ and kinetic power $W_{K}$ is around 30-70\% smaller than independent estimates obtained from observations of precipitation. This discrepancy results from the limited resolution of the MERRA database. Coarse spatial and temporal averaging causes "spurious evaporation" to occur in the large-scale descending air motions thus reducing the magnitude of all quantities - including $W_{P}$ - proportional to condensation rate (Section 5.2). 
Atmos. Chem. Phys. Discuss., doi:10.5194/acp-2016-203, 2016

Manuscript under review for journal Atmos. Chem. Phys.

Published: 5 April 2016

(c) Author(s) 2016. CC-BY 3.0 License.
Atmospheric 을

Chemistry

and Physics

Discussions

(c) (i)

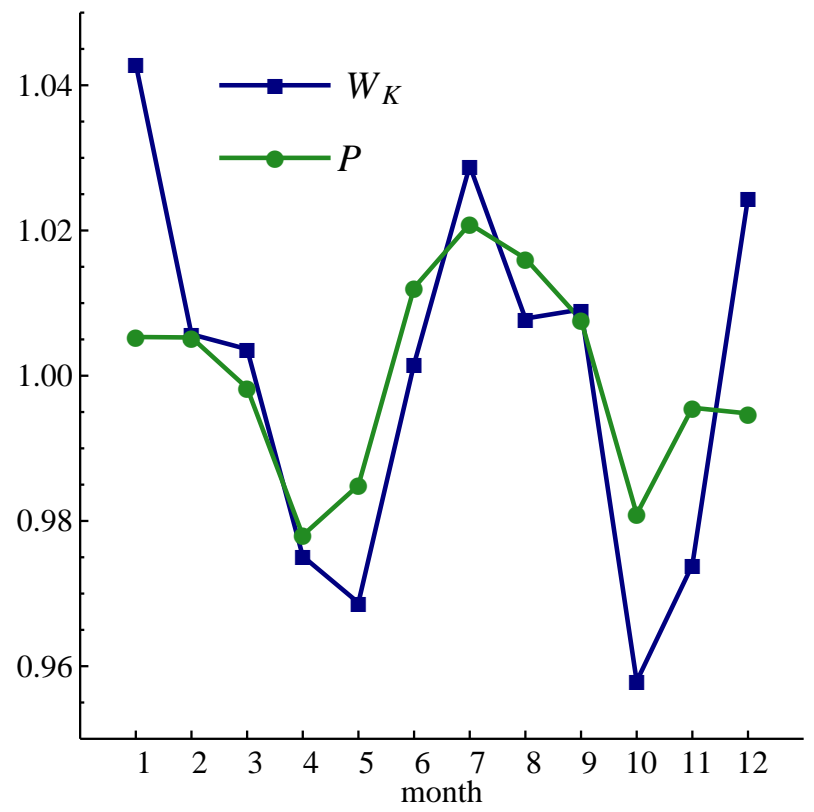

Figure 3. Mean monthly kinetic power $W_{K}$ and global mean precipitation $P$ for the time period 2009-2014. Monthly $W_{K}$ and $P$ values divided by their 2009-2014 mean values of, respectively, $2.6 \mathrm{~W} \mathrm{~m}^{-2}$ and $2.64 \mathrm{~mm} \mathrm{day}^{-1}$. The year 2015 was excluded because of incomplete precipitation data (see Fig. 1b,c).

That the data resolution has a major impact on $W_{P}$ gains further support from the analyses of the water budget in the MERRA database (Bosilovich et al., 2011). Here an atmospheric moisture budget consistent with observations cannot be retrieved from the observed wind speeds and water vapor mixing ratios alone: an additional correction ("analysis") term must be added to the mass equation for atmospheric water. This correction term is of the order of the difference between local evaporation and precipitation (Bosilovich et al., 2011), i.e. it is of the order of $\int_{0}^{z\left(p_{T}\right)} \dot{\rho} d z$ in the vertically integrated continuity equation (13). Thus $W_{P}$ cannot be retrieved from the MERRA database based on only the continuity equations (6), (13) that underlie the estimate of atmospheric power $W$.

Next we consider how our results are relevant to the problem of finding constraints on global atmospheric power.

\subsection{The upper limit}

Power output of a system cannot exceed the power output $W_{C}$ of the Carnot cycle. To quantify this limit on atmospheric power, three variables are required: input temperature $T_{i n}$, output temperature $T_{\text {out }}$ and heat flux $F$ :

$W_{C}=F \frac{\Delta T_{C}}{T_{\text {in }}}, \Delta T_{C} \equiv T_{\text {in }}-T_{\text {out }}, \quad F=F_{L}+F_{S}$.

Here $F\left(\mathrm{~W} \mathrm{~m}^{-2}\right)$ is equal to the sum of latent $F_{L}$ and sensible $F_{S}$ heat fluxes. The heat flux available to the Earth's atmospheric engine is limited by the incoming flux of solar radiation. The minimum output temperature $T_{\text {out }}=T_{E}$ is set by the Earth's 
Atmos. Chem. Phys. Discuss., doi:10.5194/acp-2016-203, 2016

Manuscript under review for journal Atmos. Chem. Phys.

albedo and orbital position: it is the temperature at which the atmosphere emits thermal radiation to space. The actual output temperature of the atmospheric engine can be higher, $T_{\text {out }} \geq T_{E}$ : in this case the part of the atmosphere that produces work would release heat not directly to space but to the upper atmospheric layers. The upper atmosphere will further transmit heat to space without generating work. The input temperature is bounded from above by temperature $T_{s}$ of the Earth's surface, $T_{i n} \leq$ $T_{s}$, and thus depends on the magnitude of the greenhouse effect $\Delta T \equiv T_{s}-T_{E}$. However, this magnitude is a priori unknown. With the Earth's extensive oceans, there is a positive feedback between surface temperature and atmospheric moisture, since this moisture is itself a major greenhouse substance. This means that the greenhouse effect on an Earth-like planet could range within broad limits. Even among the planets of the solar system the maximum Carnot efficiency $\Delta T / T_{s}$ varies at least six-fold (Schubert and Mitchell, 2013).

If we cannot predict $\Delta T$ from theory, there is only one robust theoretical limit on $W$ that we can infer from thermodynamics: $W$ cannot be larger than approximately $F\left(T_{S}-T_{E}\right) / T_{S}$, where $T_{S}$ is the Sun's temperature. This is the upper limit that is given by consideration of entropy production on the Earth. The global efficiency of solar energy conversion into useful work amounts to about 90\% (Wu and Liu (2010); see also Pelkowski (2012) for a rigorous theoretical discussion). This is about two orders of magnitude larger than the observed efficiency of atmospheric circulation, which is of the order of $1 \%$ of the incoming solar radiation. The thermodynamic theoretical upper limit alone is therefore of limited use for constraining the atmospheric power.

We need additional constraints on atmospheric power. One arises from consideration of the dynamic properties of atmospheric water vapor. The pressure of saturated water vapor is controlled by temperature (unlike temperature and molar density as occurs for any non-condensable gas). In the presence of a gravitational field, this property has important consequences: while dry air can rise adiabatically in a state infinitely close to hydrostatic equilibrium, the saturated water vapor cannot.

The resulting dynamics can be illustrated on the example of a simple system: a horizontally homogeneous atmosphere composed of pure water vapor, where there is only vertical motion, $\mathbf{v}=\mathbf{w}$. The water vapor condenses as it rises and water returns to the Earth in its solid or liquid form. In this case kinetic energy is produced per unit volume at a rate of $-w\left(\partial p_{v} / \partial z+\right.$ $\left.\rho_{v} g\right)=-w\left(\partial p_{v} / \partial z+p_{v} / \mathcal{H}_{v}\right)$. Here $\rho_{v}$ is mass density of water vapor and $\mathcal{H}_{v} \equiv R T /\left(M_{v} g\right) \approx 13 \mathrm{~km}$ is the hydrostatic scale height for water vapor (see also Makarieva et al., 2013b, 2014, and references therein). If the pressure distribution of water vapor were hydrostatic, then total power $-w \cdot \nabla p\left(\mathrm{~W} \mathrm{~m}^{-3}\right)$ would be spent to raise the potential energy of the ascending gas, leaving nothing to kinetic power. When saturated water vapor rises and cools, its partial pressure diminishes governed by decreasing temperature, the sum in braces is not zero and the hydrostatic equilibrium is not possible.

In the real atmosphere, in the presence of a sufficient amount of non-condensable gases a hydrostatic equilibrium is possible. If it is realized, the kinetic power $W_{K c}$ that derives from condensation of water vapor (which retains a non-hydrostatic 
Atmos. Chem. Phys. Discuss., doi:10.5194/acp-2016-203, 2016

Manuscript under review for journal Atmos. Chem. Phys.

Published: 5 April 2016

(c) Author(s) 2016. CC-BY 3.0 License.
Atmospheric

Chemistry

and Physics

Discussions

distribution) is generated in the horizontal plane:

$W_{K c}=-\frac{1}{\mathcal{S}} \int_{\mathcal{V}} \mathbf{u} \cdot \nabla p d \mathcal{V}=-\frac{1}{\mathcal{S}} \int_{\mathcal{V}} w\left(\frac{\partial p_{v}}{\partial z}+\frac{p_{v}}{\mathcal{H}}\right) d \mathcal{V}=-\frac{1}{\mathcal{S}} \int_{\mathcal{V}} w N R T \frac{\partial \gamma}{\partial z} d \mathcal{V} \approx \Pi R T_{c}=P g \mathcal{H}_{v}$,

$w N \frac{\partial \gamma}{\partial z} \approx \dot{N} \equiv \frac{\dot{\rho}}{M_{v}}, z>0$,

$T_{c} \equiv-\frac{1}{P \mathcal{S}} \int_{z>0} T(z) \dot{\rho} d \mathcal{V} \approx 270 \mathrm{~K}$

Here $P(22)$ and $\Pi \equiv P / M_{v}$ are global precipitation in units of $\mathrm{kg} \mathrm{m}^{-2} \mathrm{~s}^{-1}$ and $\mathrm{mol} \mathrm{m}^{-2} \mathrm{~s}^{-1}$, respectively; $\gamma \equiv p_{v} / p=N_{v} / N$, $\mathcal{H}_{v} \equiv R T_{c} /\left(M_{v} g\right), \mathcal{H} \equiv R T_{c} /(M g) ; T_{c}$ is mean temperature at which condensation occurs. Its global value $T_{c}=270 \mathrm{~K}$ is estimated in Appendix A. Details of theoretical estimate (25) were elaborated elsewhere (see Makarieva et al., 2013b, 2015a, and references therein). Here we discuss not the result per se, but its implications for understanding the atmosphere as a heat engine.

From Eqs. (20)-(22) and (25) for total power $W_{c}$ of the condensation-driven circulation we obtain

$W_{c}=W_{K c}+W_{P}=\left(1+\frac{\mathcal{H}_{P}}{\mathcal{H}_{v}}\right) \Pi R T_{c}=\left(1+\frac{\mathcal{H}_{P}}{\mathcal{H}_{v}}\right) \frac{R T_{c}}{L} F_{L}$

Here $L=45 \times 10^{3} \mathrm{~J} \mathrm{~mol}^{-1}$ is the latent heat of vaporization, $\Pi=F_{L} / L$. A remarkable property of Eq. (28) is that total power is proportional to the absolute temperature $T_{c}$ and, unlike the Carnot equation (24), is not related to any temperature difference. For cases when $\Delta T_{C} \ll T_{s} \approx T_{c}$, the two equations combined constrain $\Delta T_{C}$. Putting $W_{c}=W_{C}$ in Eqs. (28) and (24) we find

$\Delta T_{C}=T_{c}\left(\frac{R T_{s}}{L}\right) \frac{1+\mathcal{H}_{P} / \mathcal{H}_{v}}{1+F_{S} / F_{L}} \approx 15 \mathrm{~K}$

Here we used $F_{L}=85 \mathrm{~W} \mathrm{~m}^{-2}, F_{S}=19 \mathrm{~W} \mathrm{~m}^{-2}$ (Ohmura and Raschke, 2005), $\mathcal{H}_{P}=3.4 \mathrm{~km}$, see Appendix A, $\mathcal{H}_{v}=$ $R T_{c} /\left(M_{v} g\right)=12.7 \mathrm{~km}$, and $T_{s}=288 \mathrm{~K}$ as the global mean surface temperature.

This theoretical estimate of $\Delta T_{C}$ obtained under the assumption (25) that the circulation on Earth is condensation-driven coincides within $20 \%$ with an independent estimate of $\Delta T_{c} \equiv T_{s}-T_{c}=18 \mathrm{~K}$ between the surface temperature and the mean condensation temperature $T_{c}$, Eq. (A5). This consistency suggests that the condensation-driven circulation on Earth is equivalent to Carnot cycle operating between the surface temperature and the mean temperature $T_{c}=270 \mathrm{~K}$ where condensation occurs. This agrees with the observation that a major part of kinetic power is generated in the lower atmosphere, Fig. 2. The gravitational power of precipitation follows the vertical profile of the water vapor mixing ratio and is also maximum in the lower atmosphere, see Fig. 2 of Pauluis and Dias (2012) and Fig. 2 of Makarieva et al. (2013a).

The global kinetic power estimated from Eq. (25) using $T_{c}=270 \mathrm{~K}$ and $P=0.96 \mathrm{~m} \mathrm{year}^{-1}$ is $3.8 \mathrm{~W} \mathrm{~m}^{-2}$, Table 1 . This theoretical estimate is $30 \%$ higher than our estimate of $W_{K}=2.6 \mathrm{~W} \mathrm{~m}^{-2}$ obtained using the MERRA re-analysis. Similarly, theoretical estimate of the gravitational power of precipitation $W_{P}$ derived from global precipitation is about 50\% higher than its MERRA-derived estimate as the difference between $W$ and $W_{K}$ (20), Table 1. The theoretical estimate of the mass integral of $d h / d t(15)$ is also about 70\% higher (by absolute magnitude) than its MERRA-derived estimate. 
Atmos. Chem. Phys. Discuss., doi:10.5194/acp-2016-203, 2016

Atmospheric

Chemistry

Manuscript under review for journal Atmos. Chem. Phys.

Published: 5 April 2016

(c) Author(s) 2016. CC-BY 3.0 License.

and Physics

Discussions

(c) (i)

We have suggested that in the latter two cases this discrepancy can be attributed to the coarse spatial and temporal resolution of the MERRA database. Averaging air velocity and water vapor mixing ratios over areas and time periods exceeding the area and lifespan of air motions associated with condensation decreases mean condensation rate. In the result, all variables that are proportional to condensation, including $W_{P}(22)$ and the $d h / d t$ integral (15), are underestimated in the MERRA database. From this logic we can expect that with increasing spatial and temporal resolution the atmospheric power estimated from observations should grow. In agreement with these ideas, Kim and Kim (2013) using the daily averaged MERRA data obtained $W_{K}=2 \mathrm{~W} \mathrm{~m}^{-2}$, which is $20 \%$ less than the value obtained by Huang and McElroy (2015) and by us here using three hourly data. Total atmospheric power calculated with a sufficient resolution capturing the motions that cause condensation is thus likely to be around $5 \mathrm{~W} \mathrm{~m}^{-2}$, Table 1 .

The fact that $W_{K c}$ is likewise higher than our MERRA-derived kinetic power, testifies in favor of the theoretical estimate (25). To explain this point in greater detail: Eq. (15) and Eq. (22), which estimate, respectively, the mass integral of $d h / d t$ and the gravitational power of precipitation $W_{P}$, are not dependent on the assumption that air circulation on Earth is condensationdriven. These equations describe how the corresponding variables can be estimated from observations. Both variables are approximately proportional to the volume integral of net condensation rate in the atmospheric interior $-\int_{z>0} \dot{\rho} d \mathcal{V}$. We notice that both variables estimated from the MERRA database are by 50-70\% smaller than when estimated independently from the observed global precipitation $P$. We attribute this to the insufficient spatial resolution of the air motions associated with condensation. Now, we predict that if atmospheric circulation is condensation-driven, kinetic power generation is also proportional to $P$, as described by Eq. (25). Since we already know that not all condensation is resolved in the MERRA dataset, we can expect that kinetic energy generation estimated from MERRA using Eq. (21) will be smaller by a comparable magnitude than its theoretical estimate (25). This is what we find. If kinetic power was unrelated to precipitation, we could not expect that its value would be smaller than the precipitation-based theoretical estimate (25). If, on the other hand, our theoretical estimate turned out to be smaller than the MERRA-derived estimate, $W_{K c}<W_{K}$, this would testify against condensation-induced dynamics.

\subsection{The lower limit}

Why does the atmosphere generate any appreciable power at all, i.e. what determines the lower limit of $W$ ? Atmospheric power $W$ can be viewed as a measure of the dynamic disequilibrium of the Earth's atmosphere. In equilibrium, for example, under conditions of hydrostatic and geostrophic or cyclostrophic balance, no power is generated: $W=0$. There is no vertical air motion and practically no precipitation. There are no surface fluxes of sensible and latent heat. In global circulation models a non-zero rate of kinetic energy generation is achieved by introducing an ad hoc intensity of turbulent diffusion, which is chosen by fitting the model to observations. Turbulent diffusion determines the rate at which kinetic energy is dissipated (and, in the steady state, generated) (see also discussion in Makarieva et al., 2015b). It is this, and related parameterizations of dissipative processes, that postulate a certain non-zero value of $W$ in the terrestrial atmosphere and control its behavior. For example, putting turbulent diffusion in the atmospheric interior close to zero, Held and Hou (1980) described an otherwise realistic general circulation in a dry atmosphere that was about an order of magnitude less intense than observed on Earth. We find no obvious grounds to expect that the atmospheric circulation on Earth could not be significantly weaker than it is today. 
Atmos. Chem. Phys. Discuss., doi:10.5194/acp-2016-203, 2016

Atmospheric

Chemistry

Manuscript under review for journal Atmos. Chem. Phys.

Published: 5 April 2016

(c) Author(s) 2016. CC-BY 3.0 License.

and Physics

Discussions

(c) (i)

To what degree does atmospheric power depend on the Earth possessing a moist atmosphere? A moist atmosphere differs from a dry atmosphere by manifesting distinct processes that can generate air motion. One, the release of latent heat in the ascending air, has received much attention in studies of the atmospheric heat engine (e.g., Goody, 2003; Pauluis, 2011; Kleidon et al., 2014; Kieu, 2015).

Condensation-induced dynamics introduces a distinct mechanism: any upward motion of a saturated air parcel results in condensation and precipitation which diminishes local surface pressure via a hydrostatic adjustment. This leads to air convergence towards the resulting low pressure. Irrespective of whether this initial air motion gets extinguished or sustains itself via persistent condensation of laterally imported water vapor, a certain amount of kinetic energy is generated ${ }^{7}$. In a dry atmosphere this mechanism does not exist, since upward displacements of dry air do not change the amount of gas.

These condensation-related mechanisms permit self-induced air motion. Condensation occurs and the condensation-related potential energy is released as the air rises in the gravitational field of Earth. This implies a positive feedback between the motion of moist air and the release of potential energy that sustains it. In a dry atmosphere such positive feedback is absent.

One can expect that this positive feedback will drive the atmosphere to a state when it will consume all available power such that condensation rate is maximized. Such an atmosphere will be circulating in the vertical dimension with its vertical velocity constrained by the absorbed solar power and the condition of maximum precipitation and minimum net radiative and sensible heat fluxes. On Earth, precipitation accounts for a major part of the solar power absorbed so this situation is realistic. In a dry atmosphere, with such mechanisms for self-induced air motions absent, atmospheric power can remain much lower.

Whether the atmospheric power would be negligible on a dry Earth is a theoretical question. The parameterization of turbulence in current models is generally unrelated to the hydrological cycle (i.e. one and the same turbulent diffusion coefficient can be used in both dry and moist models). Therefore, comparing $W$ across current models with varying intensity of the hydrological cycle cannot clarify the role of water vapor. What is needed are theoretical insights that could be tested against observations. Direct tests are unfeasable - one cannot dry the Earth's atmosphere to see what happens. But one can study phenomena such as tropical cyclones that require condensation and see whether similar processes can clarify global atmospheric circulation (see, e.g., Makarieva et al., 2015a). One can investigate circulation power on planets with or without intense phase transitions.

Furthermore, one could re-formulate dissipative processes in the existing global circulation models such that they conform to condensation-driven dynamics and see how they perform. Currently the parameterization of dissipative processes is governed by the requirement that the observed pressure gradients must yield the observed wind velocities. Within broad limits any model, dry or moist, can be parameterized to yield any desirable rate of wind power generation/dissipation. However, if indeed wind power is linked to condensation, then models that neglect this relationship - though they may be calibrated to replicate observed wind velocities - cannot predict circulation intensity, precipitation patterns and other related phenomena under changing climatic conditions (Bony et al., 2015).

By showing that condensation-induced dynamics can readily explain the magnitude of observed wind power, our work suggests that in the absence of condensation the atmospheric power on Earth would be substantially smaller. This offers

\footnotetext{
${ }^{7}$ Whether latent heat release generates any positive atmospheric power is wholly dependent on the sufficiently rapid cooling of the descending air (Goody, 2003). Without such cooling, atmospheric power production from latent heat is impossible.
} 
Atmos. Chem. Phys. Discuss., doi:10.5194/acp-2016-203, 2016

Manuscript under review for journal Atmos. Chem. Phys.

Published: 5 April 2016

(c) Author(s) 2016. CC-BY 3.0 License.
Atmospheric

Chemistry

and Physics

Discussions

(c) (i)

important insights into regional moisture regimes and the influence of land cover. Deforestation disturbs terrestrial evaporation and resulting condensation and diminishes the soil moisture store. One possible effect is changed partitioning between smallscale atmospheric power generated on the scale of convective eddies and large-scale atmospheric power generated at continental scale. This would influence air circulation and the terrestrial water cycle. Such mechanisms may explain the shifts in rainfall recently observed in world regions affected by deforestation like Brazil and the Mediterranean (Marengo and Espinoza, 2015; Dobrovolski and Rattis, 2015; Cook et al., 2016). We urge increased attention to the dynamic effects of condensation.

\section{Appendix A: Estimating the enthalpy integral (15), $W_{P}(22)$ and $T_{c}(27)$}

We follow the approach of Makarieva et al. (2013a). We assume that moist air having temperature $T_{s}$ and relative humidity $80 \%$ at the surface first rises dry adiabatically up to height $z_{1}$ where water vapor becomes saturated. Then it rises moist adiabatically to $z_{2}$, where condensation ceases. At $z_{2}$ the air preserves share $\zeta$ of its initial water vapor content, $\zeta \equiv \gamma\left(z_{2}\right) / \gamma_{s}=\gamma\left(z_{2}\right) / \gamma\left(z_{1}\right)$. Here $\gamma \equiv p_{v} / p$, where $p_{v}$ is water vapor partial pressure and $p$ is air pressure. Moist adiabatic distributions of $\gamma(z), T(z)$ and $p(z)$ with $p_{s}=1000 \mathrm{hPa}$ were calculated according to Eqs. (A3)-(A5) of Makarieva et al. (2013a).

For $T_{s}$ ranging from 260 to $310 \mathrm{~K}$ and for $\zeta$ ranging from 0.001 (complete condensation) to $3 / 4$, we estimated mean condensation height $\mathcal{H}_{P}$, mean condensation temperature $T_{c}$ and mean condensation enthalpy per mole $h_{c}$, Fig. 4 and Table 2 :

$\mathcal{H}_{P}\left(T_{s}, \zeta\right)=\frac{1}{\gamma\left(z_{2}\right)-\gamma\left(z_{1}\right)} \int_{z_{1}}^{z_{2}} z \frac{\partial \gamma}{\partial z} d z$

$T_{c}\left(T_{s}, \zeta\right)=\frac{1}{\gamma\left(z_{2}\right)-\gamma\left(z_{1}\right)} \int_{z_{1}}^{z_{2}} T(z) \frac{\partial \gamma}{\partial z} d z, \quad \Delta T_{c} \equiv T_{s}-T_{c}$,

$h_{c}\left(T_{s}, \zeta\right)=\frac{1}{\gamma\left(z_{2}\right)-\gamma\left(z_{1}\right)} \int_{z_{1}}^{z_{2}} h(z) \frac{\partial \gamma}{\partial z} d z, \quad \Delta h_{c} \equiv h_{s}-h_{c}, \quad h(z)=c_{p} T(z)+L \gamma(z), \quad c_{p}=(7 / 2) R$

To find the corresponding global mean values we consider the tropics (the area between $30^{\circ} \mathrm{S}$ and $30^{\circ} \mathrm{N}$ ) and the extratropics separately. The two regions have equal areas. The mean annual temperatures for 2009-2014 at $1000 \mathrm{hPa}$ are $296.5 \mathrm{~K}$ and $277 \mathrm{~K}$ for the tropics and the extratropics, respectively. Most tropical rainfall is associated with temperatures above $299 \mathrm{~K}$ (Johnson and Xie, 2010; Sabin et al., 2013), so we take $T_{s t}=300 \mathrm{~K}$ as a representative value for tropical rainfall. In the extratropics there is also a tendency for higher rainfall at higher temperature, Fig. 5, we take $T_{s e}=280 \mathrm{~K}$. According to the Global Precipitation Climatology Project (GPCP) version 2.2 dataset, tropical and extratropical precipitation in 2009-2014 was, respectively, $P_{t}=3.03 \mathrm{~mm}$ day $^{-1}$ and $P_{e}=2.24 \mathrm{~mm}$ day ${ }^{-1}$. We estimated mean global values of $\mathcal{H}_{P}, \Delta T_{c}$ and $\Delta h_{c}$ from Eqs. (A1)-(A3) as

$X(\zeta)=\frac{X\left(T_{s t}, \zeta\right) P_{t}+X\left(T_{s e}, \zeta\right) P_{e}}{P_{t}+P_{e}}$.

The results are shown in Table 2. 
Atmos. Chem. Phys. Discuss., doi:10.5194/acp-2016-203, 2016

Manuscript under review for journal Atmos. Chem. Phys.

Published: 5 April 2016

(c) Author(s) 2016. CC-BY 3.0 License.

(c) (i)
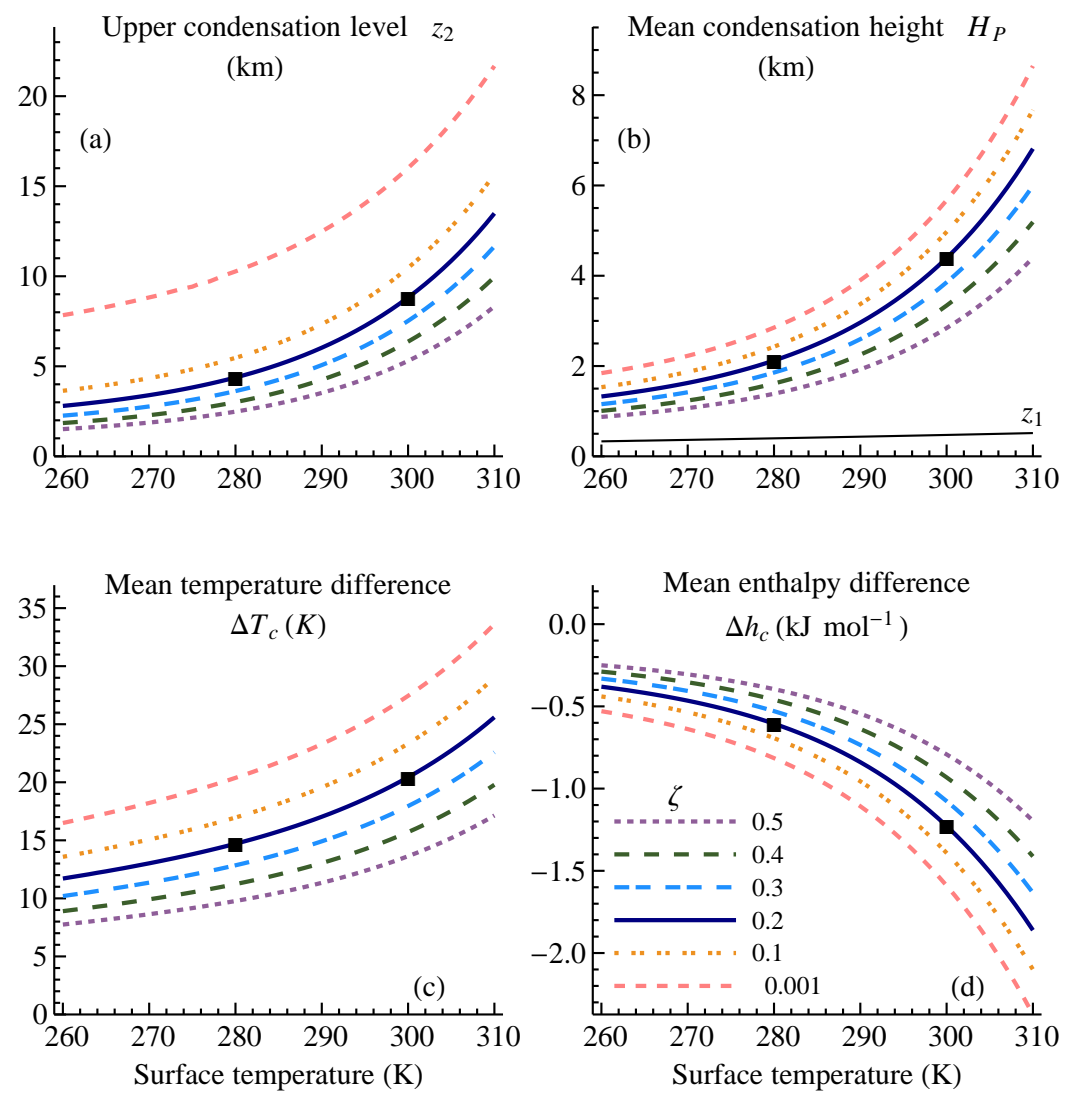

Figure 4. The upper condensation level $z_{2}$ (a), lower condensation level $z_{1}$ and $\mathcal{H}_{P}$ (A1) (b), $\Delta T_{c}$ (A2) (c) and $\Delta h_{c}$ (A3) (d) as dependent on surface temperature $T_{s}$ and incompleteness of condensation $\zeta$. Solid squares show values used for the global mean estimates (A5).

Assuming that tropical $W_{P t}=1.5 \mathrm{~W} \mathrm{~m}^{-2}$ according to the TRMM measurements analyzed by Pauluis and Dias (2012), we conclude from Table 2 that under our assumptions the results with $\zeta>0.4$ (more than $40 \%$ of water vapor does not condense) corresponding to the global mean $W_{P}<0.75 \mathrm{~W} \mathrm{~m}^{-2}$ are not realistic. This is smaller than half the tropical average and is thus impossible. For the same reason our previous estimate $W_{P}=0.8 \mathrm{~W} \mathrm{~m}^{-2}$, which corresponds to a negligible contribution from the extratropical rainfall to total gravitational power, appears an underestimate ${ }^{8}$. The tropical estimate $W_{P t}$ coincides with the TRMM-derived estimate of Pauluis and Dias (2012) for $\zeta=0.2$. In this case $W_{P}=1 \mathrm{~W} \mathrm{~m}^{-2}$. We will thus use the

\footnotetext{
${ }^{8}$ The estimate of $W_{P}=0.8 \mathrm{~W} \mathrm{~m}^{-2}$ was obtained by Makarieva et al. (2013a) from Eq. (22) assuming that $\mathcal{H}_{P}=2.5 \mathrm{~km}$ is a representative value for the global average. This height corresponds to the following case: at the surface the ascending air has a global mean surface temperature $288 \mathrm{~K}$ and relative humidity $80 \%$; above the point of saturation it rises with mean tropospheric lapse rate $6.5 \mathrm{~K} \mathrm{~km}^{-1}$; about one quarter of the water vapor does not condense and remains in the ascending air, i.e. $\zeta=1 / 4$.
} 
Atmos. Chem. Phys. Discuss., doi:10.5194/acp-2016-203, 2016

Manuscript under review for journal Atmos. Chem. Phys.

Published: 5 April 2016

(c) Author(s) 2016. CC-BY 3.0 License.

(c) (i)
Atmospheric

Chemistry

and Physics

Discussions
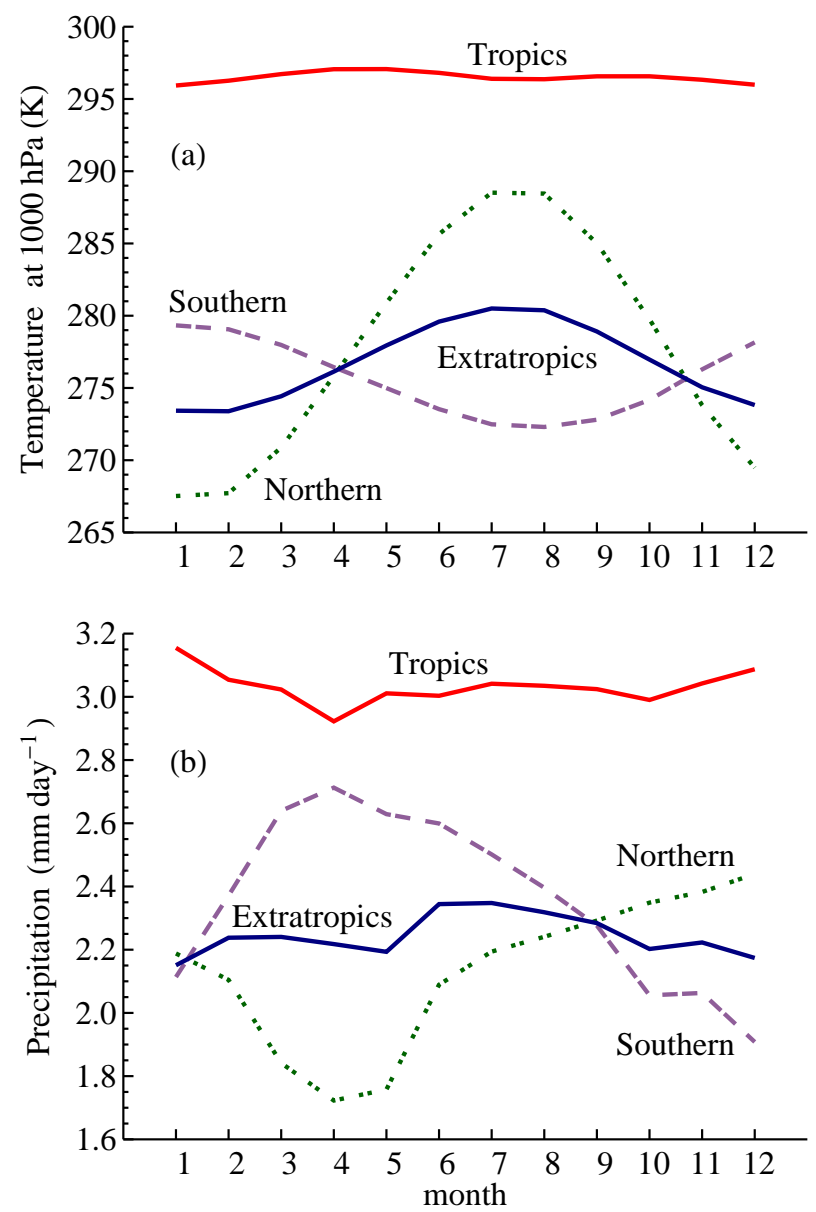

Figure 5. Mean monthly temperature at $1000 \mathrm{hPa}$ (a) and GPCP v. 2.2 precipitation (b) in $2009-2014$ in the tropics $\left(30^{\circ} \mathrm{S}-30^{\circ} \mathrm{N}\right)$ and the extratropics $\left(90^{\circ} \mathrm{S}-30^{\circ} \mathrm{S}\right.$ and $\left.30^{\circ} \mathrm{N}-90^{\circ} \mathrm{N}\right)$.

case $\zeta=0.2$ as a representative value for the global mean, Table 2 :

$\zeta=0.2, \quad \Delta T_{c}=18 \mathrm{~K}, \quad-P \Delta h_{c}=-1.6 \mathrm{~W} \mathrm{~m}^{-2}, \quad W_{P}=1 \mathrm{~W} \mathrm{~m}^{-2}$.

Note that in the interval $0 \leq \zeta \leq 0.3$ all the values in Table 2 change about 1.3 -fold, which suggests that the uncertainty of the global values should be under $30 \%$.

\section{Appendix B: Details of calculating $W$ and $W_{K}$}

The MERRA dataset MAI3CPASM version 5.2.0 was downloaded for the years 2009-2015 (it contains one file for each day) from http://mirador.gsfc.nasa.gov. We chose this dataset because it contained the pressure velocity $\omega$ necessary for calculating total atmospheric power. The data are provided for eight times of the day $(t=1, \ldots, 8): 00,03,06,09,12,15,18$ and 21 hours. 
Atmos. Chem. Phys. Discuss., doi:10.5194/acp-2016-203, 2016

Manuscript under review for journal Atmos. Chem. Phys.

Chemistry

Published: 5 April 2016

(c) Author(s) 2016. CC-BY 3.0 License.

Table 2. Global mean estimates of the mean condensation height $\mathcal{H}_{P}$, mean condensation temperature difference $\Delta T_{c}$ and enthalpy difference $\Delta h_{c}$ (A4) and the global gravitational power of precipitation $W_{P}$ dependent on $\zeta$ (incompleteness of condensation). Subscripts $t$ and $e$ refer to corresponding values in the tropics and extratropics. Boldfaced line shows values for $\zeta=0.2$ which are consistent with TRMM-derived estimate for tropical $W_{P t}=1.5 \mathrm{~W} \mathrm{~m}^{-2}$ obtained by Pauluis and Dias (2012). Note that $P=\left(P_{t}+P_{e}\right) / 2$ and $W_{P}=\left(W_{P t}+W_{P e}\right) / 2$.

\begin{tabular}{rllllllllllll}
\hline \multicolumn{1}{c}{$\zeta$} & $\mathcal{H}_{P t}$ & $\mathcal{H}_{P e}$ & $\mathcal{H}_{P}$ & $\Delta T_{c t}$ & $\Delta T_{c e}$ & $\Delta T_{c}$ & $-P_{t} \Delta h_{c t}$ & $-P_{e} \Delta h_{c e}$ & $-P \Delta h_{c}$ & $W_{P t}$ & $W_{P e}$ & $W_{P}$ \\
& $\mathrm{~km}$ & $\mathrm{~km}$ & $\mathrm{~km}$ & $\mathrm{~K}$ & $\mathrm{~K}$ & $\mathrm{~K}$ & $\mathrm{~W} \mathrm{~m}^{-2}$ & $\mathrm{~W} \mathrm{~m}^{-2}$ & $\mathrm{~W} \mathrm{~m}^{-2}$ & $\mathrm{~W} \mathrm{~m}^{-2}$ & $\mathrm{~W} \mathrm{~m}^{-2}$ & $\mathrm{~W} \mathrm{~m}^{-2}$ \\
\hline 0.00 & 5.7 & 2.9 & 4.5 & 27.4 & 20.4 & 24.4 & -3.10 & -1.17 & -2.14 & 1.95 & 0.72 & 1.34 \\
0.10 & 5.0 & 2.4 & 3.9 & 23.3 & 17.0 & 20.6 & -2.71 & -1.00 & -1.85 & 1.71 & 0.62 & 1.16 \\
$\mathbf{0 . 2 0}$ & $\mathbf{4 . 4}$ & $\mathbf{2 . 1}$ & $\mathbf{3 . 4}$ & $\mathbf{2 0 . 4}$ & $\mathbf{1 4 . 7}$ & $\mathbf{1 8 . 0}$ & $\mathbf{- 2 . 3 9}$ & $-\mathbf{0 . 8 7}$ & $-\mathbf{1 . 6 3}$ & $\mathbf{1 . 5 1}$ & $\mathbf{0 . 5 4}$ & $\mathbf{1 . 0 2}$ \\
0.25 & 4.1 & 2.0 & 3.2 & 19.2 & 13.7 & 16.9 & -2.24 & -0.82 & -1.53 & 1.42 & 0.50 & 0.96 \\
0.30 & 3.9 & 1.9 & 3.0 & 17.9 & 12.8 & 15.8 & -2.10 & -0.76 & -1.43 & 1.32 & 0.47 & 0.90 \\
0.40 & 3.3 & 1.6 & 2.6 & 15.7 & 11.2 & 13.8 & -1.82 & -0.66 & -1.24 & 1.15 & 0.41 & 0.78 \\
0.50 & 2.8 & 1.4 & 2.2 & 13.6 & 9.8 & 12.0 & -1.54 & -0.57 & -1.06 & 0.98 & 0.35 & 0.66 \\
0.60 & 2.4 & 1.2 & 1.9 & 11.7 & 8.4 & 10.3 & -1.28 & -0.48 & -0.88 & 0.81 & 0.30 & 0.55 \\
0.70 & 1.9 & 1.0 & 1.5 & 9.8 & 7.2 & 8.7 & -1.02 & -0.40 & -0.71 & 0.64 & 0.25 & 0.45 \\
0.75 & 1.6 & 0.9 & 1.3 & 8.9 & 6.6 & 7.9 & -0.89 & -0.36 & -0.62 & 0.56 & 0.22 & 0.39 \\
\hline
\end{tabular}

The latitude/longitude grid has a resolution of $1.25^{\circ}$. Latitude coordinate of the grid cell center spans from $-90+1.25 / 2$ to $90-1.25 / 2$ degrees Northern latitude $(i=1, \ldots, 144)$. Longitude coordinate of the grid cell center spans from $-180+1.25 / 2$ to $180-1.25 / 2$ degrees Eastern longitude $(j=1, \ldots, 288)$. The vertical dimension is represented by 42 fixed pressure levels $(k=1, \ldots, 42)$, from $p_{1}=1000 \mathrm{hPa}$ to $p_{42}=0.1 \mathrm{hPa}(1000,975,950,925,900,875,850,825,800,775,750,725,700,650$, $600,550,500,450,400,350,300,250,200,150,100,70,50,40,30,20,10,7,5,4,3,2,1,0.7,0.5,0.4,0.3,0.1 \mathrm{hPa})$. For each day in the studied years we used the following variables $X_{k}(t, i, j)$ : geopotential height $H$, meridional and zonal velocity $v$ and $u$, pressure velocity omega $\omega$, temperature $T$ and the mass fraction of water vapor $q_{v} \equiv \rho_{v} / \rho$. We also used surface pressure $p_{s}(t, i, j)$. To calculate $\partial X / \partial t$ for time $t$ in a given grid cell we used the next (i.e. 3 hours after the considered time point) and the previous (3 hours before) $X$ values and divided their difference by $\Delta t=6 \mathrm{hr}$.

\section{B1 Calculation of $W$}

The procedure is best illustrated using an example. For example, we are interested in the time point $15.00(t=6)$ on 1 July 2010 for a grid cell with numbers $i=80$ (latitude) and $j=100$ (longitude). This grid cell is centered at $9.375^{\circ}$ Northern latitude and $-55.625^{\circ}$ Eastern longitude and has an area of $S(i)=1.906 \times 10^{10} \mathrm{~m}^{2}$. The atmospheric column is composed of elementary 
Atmos. Chem. Phys. Discuss., doi:10.5194/acp-2016-203, 2016

Manuscript under review for journal Atmos. Chem. Phys.

Published: 5 April 2016

(c) Author(s) 2016. CC-BY 3.0 License.
Atmospheric

Chemistry

and Physics

Discussions

volumes $\Delta \mathcal{V}_{k}$ that are enclosed by the neighboring pressure levels $k$ and $k+1$ :

$\Delta \mathcal{V}_{k}(t, i, j)=S(i)\left[H_{k+1}(t, i, j)-H_{k}(t, i, j)\right]$

where $H_{k}(t, i, j)$ is the geopotential height of the $k$-th pressure level. For example, for $k=1$ (pressure level $p_{1}=1000 \mathrm{hPa}$ ) we have in the time and place of interest $H_{1}=125 \mathrm{~m}, H_{2}=348 \mathrm{~m}$ and $\Delta \mathcal{V}_{1}=4.25 \times 10^{12} \mathrm{~m}^{3}$. The omega value corresponding to each elementary volume $\Delta \mathcal{V}_{k}$ (B1) was calculated as $\omega=\left(\omega_{k}+\omega_{k+1}\right) / 2$, i.e. as the average of the omega values at the neighboring pressure levels defining the elementary volume.

We also need to calculate the contribution of the near surface layer that is enclosed between the pressure levels $p_{s}$ (surface pressure) and $p_{k_{\text {min }}}$, where $k_{\min }(t, i, j)$ is the number of the level with maximum pressure for which the data exists for a given grid cell and time point. For example, in mountainous areas there are no atmospheric layers with $k=1$ and $p_{1}=1000 \mathrm{hPa}$ in such areas $k_{\min }>1$.

To find the vertical thickness $\Delta z$ of the surface layer we used the hydrostatic equation $\partial p / \partial z=-p / \mathcal{H}$, where $\mathcal{H}=R T / M g$ is the local exponential scale height for air pressure. We estimated the elementary volume $\Delta \mathcal{V}_{s}(t, i, j)$ in the surface layer as

$\Delta \mathcal{V}_{s}=S(i) \Delta z=S(i) \frac{p_{s}-p_{k_{\text {min }}}}{p_{k_{\text {min }}}} \mathcal{H}_{k_{\text {min }}}, \quad \mathcal{H}_{k} \equiv \frac{R T_{k}}{M_{k} g}, \quad M_{k} \equiv \frac{M_{d}}{1+\left(M_{d} / M_{v}-1\right) q_{v k}}$

Here $M_{d}=0.0289 \mathrm{~kg} \mathrm{~mol}^{-1}$ and $M_{v}=0.018 \mathrm{~kg} \mathrm{~mol}^{-1}$ are molar masses of dry air and water vapor, respectively. For our cell $(t=6, i=80, j=100)$ we have $k_{m i n}=1, p_{k_{m i n}}=p_{1}=10^{5} \mathrm{~Pa}, p_{s}=101409 \mathrm{~Pa}, q_{v 1}=0.0179, M_{1}=0.0286 \mathrm{~kg}$ mol -1 , $T_{1}=299.2 \mathrm{~K}$ and $\Delta \mathcal{V}_{s}=2.38 \times 10^{12} \mathrm{~m}^{3}$.

We now need to find the omega value at the surface $\omega_{s}$. (While there are surface data in the MERRA database, they are provided with a different spatial resolution than in MAI3CPASM.) This can be done in two ways, which should give identical results in the limit of infinitely small elementary volumes, $\Delta \mathcal{V}_{k} \rightarrow 0$, but different results for finite $\Delta \mathcal{V}$. The first way is to extrapolate the omega dependence on pressure linearly to the surface assuming that the derivative of omega over pressure does not change from the surface to the $\left(k_{\min }+1\right)$-th layer:

$\frac{\omega_{s}-\omega_{k_{m i n}}}{p_{s}-p_{k_{m i n}}}=\frac{\omega_{k_{m i n}+1}-\omega_{k_{m i n}}}{p_{k_{m i n}+1}-p_{k_{m i n}}}$

The second way is to assume that at the surface wind velocity is zero, such that $\nabla \cdot \mathbf{v}=0$ and omega is by definition equal to surface pressure tendency, see Eq. (23):

$\omega_{s}=\frac{\partial p_{s}}{\partial t}$

Finally, the integral of omega over the atmospheric column in each grid cell is given by

$\Delta \mathcal{V}_{s}(t, i, j) \frac{\omega_{s}(t, i, j)+\omega_{k_{\text {min }}}(t, i, j)}{2}+\sum_{k=k_{\min }}^{41} \Delta \mathcal{V}_{k}(t, i, j) \frac{\omega_{k}(t, i, j)+\omega_{k+1}(t, i, j)}{2}$

The global integral of omega for a given time point $t$ was found as the sum of Eq. (B5) over all grid cells:

$\Omega(t) \equiv-\frac{1}{\mathcal{S}} \int_{\mathcal{V}} \omega(t, z, y, x) d \mathcal{V} \equiv-\frac{1}{\mathcal{S}} \sum_{i=1}^{144} \sum_{j=1}^{288}\left(\Delta \mathcal{V}_{s} \frac{\omega_{s}+\omega_{k_{m i n}}}{2}+\sum_{k=k_{m i n}}^{41} \Delta \mathcal{V}_{k} \frac{\omega_{k}+\omega_{k+1}}{2}\right)$ 
Atmos. Chem. Phys. Discuss., doi:10.5194/acp-2016-203, 2016

Manuscript under review for journal Atmos. Chem. Phys.

Published: 5 April 2016

(c) Author(s) 2016. CC-BY 3.0 License.

Table 3. Annual mean atmospheric power $\left(\mathrm{W} \mathrm{m}^{-2}\right)$ in $2009-2015$.

\begin{tabular}{lccccccc}
\hline Variable & 2009 & 2010 & 2011 & 2012 & 2013 & 2014 & 2015 \\
\hline$W_{K 1}$ & 2.65 & 2.67 & 2.65 & 2.67 & 2.68 & 2.75 & 2.80 \\
$W_{K 2}$ & 2.49 & 2.50 & 2.49 & 2.51 & 2.52 & 2.59 & 2.63 \\
$W_{K} \equiv\left(W_{K 1}+W_{K 2}\right) / 2$ & 2.57 & 2.59 & 2.57 & 2.59 & 2.60 & 2.67 & 2.72 \\
$W_{1}$ & 3.00 & 2.96 & 2.92 & 2.94 & 2.93 & 2.98 & 3.08 \\
$W_{2}$ & 3.25 & 3.25 & 3.18 & 3.22 & 3.22 & 3.26 & 3.34 \\
$W \equiv\left(W_{1}+W_{2}\right) / 2$ & 3.12 & 3.10 & 3.05 & 3.08 & 3.07 & 3.12 & 3.21 \\
\hline
\end{tabular}

The values of $\Omega_{1}$ and $\Omega_{2}$ in Fig. 1 were obtained from Eq. (B6) with $\omega_{s}$ estimated from, respectively, Eq. (B3) and Eq. (B4),

Table 3 .

To calculate time derivative $\partial p / \partial t$ at geopotential height $H_{k}$ corresponding to the $k$-th pressure level, we used the hydrostatic equation in the form

$\left(\frac{\partial p}{\partial t}\right)_{H_{k}}=-\frac{\partial H_{k}}{\partial t} \frac{\partial p}{\partial z}=\frac{\partial H_{k}}{\partial t} \frac{p}{\mathcal{H}_{k}}$.

For the global integral we have similar to Eq. (B6):

$\Psi \equiv \frac{1}{\mathcal{S}} \int_{\mathcal{V}} \frac{\partial p}{\partial t} d \mathcal{V}=\frac{1}{\mathcal{S}} \sum_{i=1}^{144} \sum_{j=1}^{288}\left\{\frac{\Delta \mathcal{V}_{s}}{2}\left[\frac{\partial p_{s}}{\partial t}+\left(\frac{\partial p}{\partial t}\right)_{H_{k_{m i n}}}\right]+\sum_{k=k_{\min }}^{41} \frac{\Delta \mathcal{V}_{k}}{2}\left[\left(\frac{\partial p}{\partial t}\right)_{H_{k}}+\left(\frac{\partial p}{\partial t}\right)_{H_{k+1}}\right]\right\}$.

\section{B2 Calculation of $W_{K}$}

We calculated zonal and meridional pressure gradients at pressure level $k$ as follows:

$\left(\frac{\partial p}{\partial x}\right)_{k}=\left(\frac{\partial p}{\partial z}\right)_{k} \frac{\partial H_{k}}{\partial x}=\frac{p_{k}}{\mathcal{H}_{k}} \frac{\partial H_{k}}{\partial x},\left(\frac{\partial p}{\partial y}\right)_{k}=\frac{p_{k}}{\mathcal{H}_{k}} \frac{\partial H_{k}}{\partial y}$,

$\frac{\partial H_{k}(t, i, j)}{\partial x}=\frac{H_{k}(t, i, j+1)-H_{k}(t, i, j-1)}{2 \times 1.25 \times L(i)}, \frac{\partial H_{k}(t, i, j)}{\partial y}=\frac{H_{k}(t, i+1, j)-H_{k}(t, i-1, j)}{2 \times 1.25 \times L_{p}}$,

where $L(i)$ is the length of 1 degree arc along the parallel at the corresponding latitude, $L_{p}=111.127 \mathrm{~m}$ is the length of one degree arch along the meridian.

Kinetic energy generation $K_{k}$ per unit volume $\left(\mathrm{W} \mathrm{m}^{-3}\right)$ at pressure level $k$ is calculated from (B9) and (B10)

$K_{k}(t, i, j) \equiv-u_{k}(t, i, j)\left(\frac{\partial p}{\partial x}\right)_{k}-v_{k}(t, i, j)\left(\frac{\partial p}{\partial y}\right)_{k}$.

The value of $K_{s}$ at the surface is found in two ways, one by analogy with Eq. (B3):

$\frac{K_{s}-K_{k_{m i n}}}{p_{s}-p_{k_{m i n}}}=\frac{K_{k_{m i n}+1}-K_{k_{m i n}}}{p_{k_{m i n}+1}-p_{k_{m i n}}}$, 
Atmos. Chem. Phys. Discuss., doi:10.5194/acp-2016-203, 2016

Manuscript under review for journal Atmos. Chem. Phys.

$K_{s}=0$.

For the global integral $W_{K}$ for a given time point $t$ we have:

$W_{K}(t) \equiv-\frac{1}{\mathcal{S}} \int_{\mathcal{V}}(\mathbf{u} \cdot \nabla p) d \mathcal{V} \equiv \frac{1}{\mathcal{S}} \sum_{i=2}^{143} \sum_{j=1}^{288}\left(\Delta \mathcal{V}_{s} \frac{K_{s}(t, i, j)+K_{k_{\min }}(t, i, j)}{2}+\sum_{k=k_{\min }}^{41} \Delta \mathcal{V}_{k} \frac{K_{k}(t, i, j)+K_{k+1}(t, i, j)}{2}\right)$.

The values of $W_{1}$ and $W_{2}$ in Fig. 1 were obtained from Eq. (B14) with $K_{s}$ estimated from, respectively, Eq. (B12) and Eq. (B13), Table 3.

\section{Appendix C: Volume integral of pressure tendency}

As noted in Section 4, any magnitudes related to vertical velocity, including total atmospheric power $W$ (20), are associated with significant uncertainty. The vertical velocity is usually small compared to horizontal velocity. Rather than being observed directly, the vertical velocity is estimated from the generally larger horizontal velocities using the continuity equation. Minor uncertainties in the horizontal components permit major uncertainties in the vertical components.

Pressure velocity (23), which depends on vertical velocity, is calculated using the additional assumption of hydrostatic equilibrium from the continuity equation in the following form:

$\nabla_{p} \cdot \mathbf{u}+\frac{\partial \omega}{\partial p}=0, \omega(p)=-\int_{p_{s}}^{p}\left(\nabla_{p^{\prime}} \cdot \mathbf{u}\right) d p^{\prime}+\omega\left(p_{s}\right)$

Here subscript $p$ at the nabla operator indicates that it is evaluated at constant pressure. For details see, for example, Kasahara (1974, his Eq. 6.4).

Pressure velocity calculated from Eq. (C1) is apparently not equal to the actual material derivative of pressure $d p / d t(5)$. This can be illustrated with the following simple example. Consider a dry axisymmetric uniformly heated hydrostatic non-rotating atmosphere, which experiences slow periodic cooling and warming. In such an atmosphere the surface pressure tendency $\partial p_{s} / \partial t$ is always zero (because the amount of gas does not change) and horizontal velocity $\mathbf{u}$ is also zero (because of the spherical symmetry), so $\omega\left(p_{s}\right)=0$. Therefore, according to Eq. (C1), omega must be zero at all heights and at all times.

However, it is clear that for any height $z>0$ the instantaneous pressure tendency (and hence the material derivative of pressure) is not zero: it must reflect the temperature variation. In the simplest case when $p(z)=p_{s} \exp (-z / \mathcal{H})$, where $\mathcal{H}=$ $R T /(M g)$ is independent of $z$ (an isothermal atmosphere), we have $\partial p / \partial t=p\left(z / \mathcal{H}^{2}\right) \partial \mathcal{H} / \partial t$. In such an atmosphere the volume integral of $\partial p / \partial t=d p / d t$ is positive when the atmosphere is warming, and negative when it is cooling:

$\Psi=\int_{0}^{\infty} \frac{\partial p}{\partial t} d z=p_{s} \frac{\partial \mathcal{H}}{\partial t}=p_{s} \mathcal{H} \frac{1}{T} \frac{\partial T}{\partial t}$.

We calculated the global integral of pressure tendency $\Psi$ (B8) for the year 2010. It is shown in Fig. 6a together with $\Omega$ (23), (B6) and $W_{K}$ (21). We can see that $\Psi$ does indeed reflect the change of global temperature, Fig. 6b. By absolute 
Atmos. Chem. Phys. Discuss., doi:10.5194/acp-2016-203, 2016

Manuscript under review for journal Atmos. Chem. Phys.

Published: 5 April 2016

(c) Author(s) 2016. CC-BY 3.0 License.
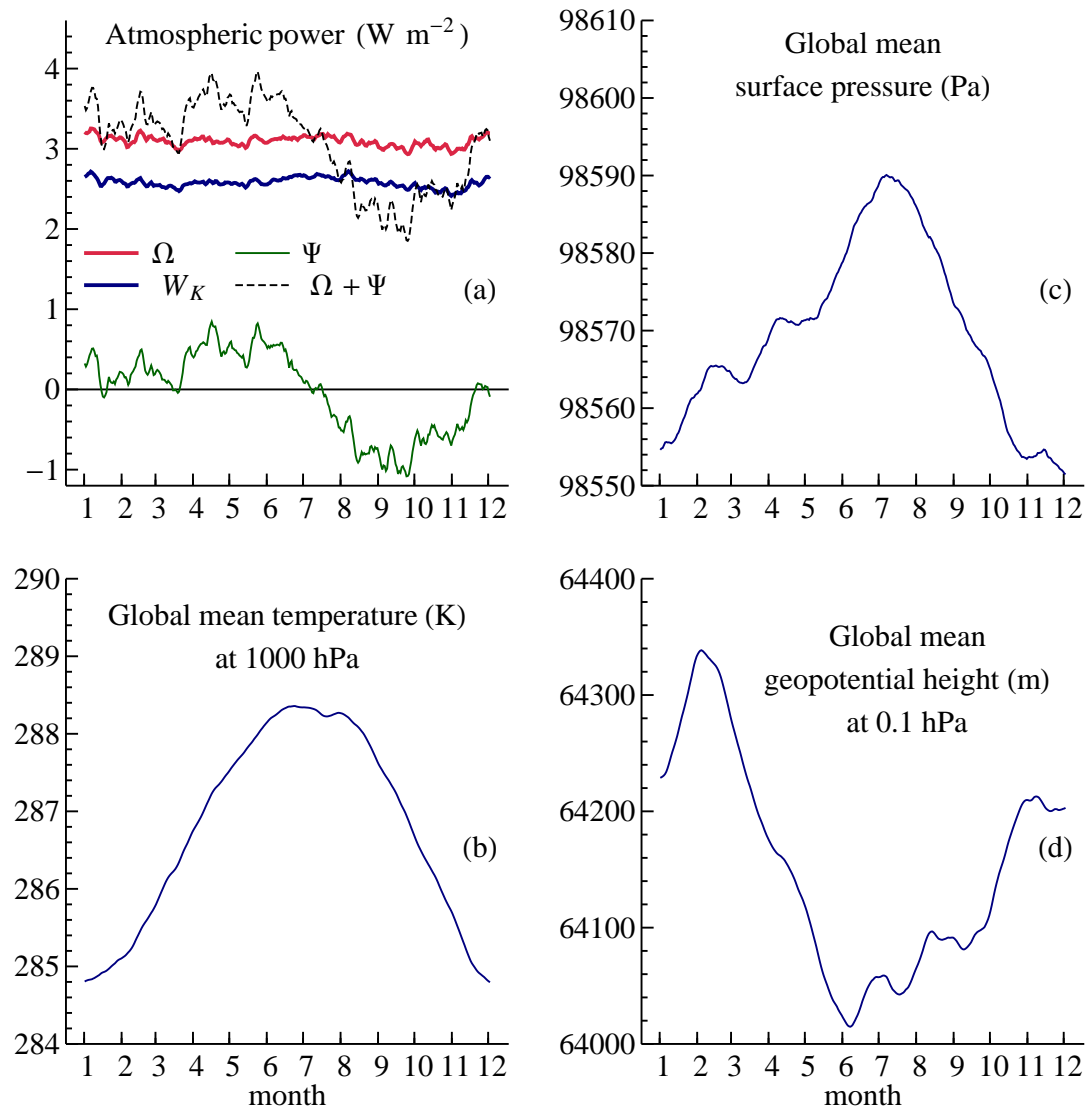

Figure 6. Time series (30-day running mean of daily values for the year 2010) of (a) the global integral of the pressure tendency $\Psi$ (B8), the omega integral (23) and kinetic power $W_{K}$ (21), cf. Fig. 1; global mean surface temperature (b), global mean surface pressure (c) and global mean geopotential height at $p_{T}=0.1 \mathrm{hPa}(\mathrm{d})$. This pressure level moves with vertical velocity $w_{T}$ of about $300 \mathrm{~m}$ in half a year, $w_{T} \sim 2 \times 10^{-5} \mathrm{~m} \mathrm{~s}^{-1}$, which corresponds to $I_{S} \sim p_{T} w_{T} \sim 10^{-4} \mathrm{~W} \mathrm{~m}^{-2} \ll W$ in Eqs. (8) and (16). Ticks on the horizontal axes correspond to the 15 th day of each month.

magnitude, $\Psi$ constitutes a considerable part (about one quarter) of total long-term atmospheric power $W$ estimated from pressure velocity. This magnitude is readily derived from Eq. (C2) for a slowly warming/cooling atmosphere. Global mean temperature $T$ changes by $3^{\circ}$ or by about $1 \%$ in half a year, $(1 / T)(\partial T / \partial t) \sim 6 \times 10^{-10} \mathrm{~s}^{-1}$, Fig. $6 \mathrm{~b}$. Global mean surface pressure changes insignificantly (by about $0.04 \%$ ) over the same period, Fig. $6 \mathrm{c}$. So during the warming phase (the first half of the year) with $p_{s}=10^{5} \mathrm{~Pa}$ and $\mathcal{H}=10^{4} \mathrm{~m}$ we obtain $\Psi=0.6 \mathrm{~W} \mathrm{~m}^{-2}$. This agrees well with Fig. $6 \mathrm{a}$.

If we formally added the integral of pressure tendency to the integral of omega, see Eq. (16), we would have obtained an absurd result whereby at certain times of the year total power would have been smaller than kinetic power, Fig. 6a, dashed curve. 
Atmos. Chem. Phys. Discuss., doi:10.5194/acp-2016-203, 2016

Manuscript under review for journal Atmos. Chem. Phys.

Published: 5 April 2016

(c) Author(s) 2016. CC-BY 3.0 License.

(c) (i)
Atmospheric

Chemistry

and Physics

Discussions

This illustrates that omega includes only those contributions from the pressure tendency that are associated with macroscopic air motions and thus non-zero gradients of horizontal velocity, Eq. (C1).

Since the long-term average of $\Psi$ is zero (for the year 2010 we have $\Psi=0.017 \mathrm{~W} \mathrm{~m}^{-2} \ll \mathrm{W}$ ), it does not affect the longterm mean estimate of $W$. However, this term can be important for quantifying conversion rates of the available potential energy to kinetic energy. Different means of calculating pressure tendency, either via Eq. (C1) or via temperature tendency Eq. (B8) should give different results for these rates (see,e.g., Kim and Kim, 2013).

Generally, Fig. 6 shows that instantaneous values of $\Omega$ do not reflect the instantaneous values of global atmospheric power $W$. Consequently, the difference $\Omega-W_{K}$ is not equal to the instantaneous value of the gravitational power of precipitation $W_{P}$. This explains why they are not correlated in Fig. $1 \mathrm{~b}$.

Author contributions. All the authors designed the research, performed the research, discussed the results and wrote the paper. A.M.M., V.G.G. and A.V.N. performed the MERRA calculations.

Acknowledgements. We thank Silvio N. Figueroa, Paulo Nobre and Rémi Tailleux for useful comments. MERRA data used in this study/project have been provided by the Global Modeling and Assimilation Office (GMAO) at NASA Goddard Space Flight Center through the NASA GES DISC online archive. This work is partially supported by Russian Scientific Foundation under Grant no. 14-22-00281 and the University of California Agricultural Experiment Station. DS's contribution denotes an output of the Australian Research Council project DP160102107. 
Atmos. Chem. Phys. Discuss., doi:10.5194/acp-2016-203, 2016

Manuscript under review for journal Atmos. Chem. Phys.

Published: 5 April 2016

(c) Author(s) 2016. CC-BY 3.0 License.
Atmospheric

Chemistry

and Physics

Discussions

\section{References}

Adler, R. F., Huffman, G. J., Chang, A., Ferraro, R., Xie, P.-P., Janowiak, J., Rudolf, B., Schneider, U., Curtis, S., Bolvin, D., Gruber, A., Susskind, J., Arkin, P., and Nelkin, E.: The Version-2 Global Precipitation Climatology Project (GPCP) Monthly Precipitation Analysis (1979-Present), J. Hydrometeor., 4, 1147-1167, doi:10.1175/1525-7541(2003)004<1147:TVGPCP>2.0.CO;2, 2003.

Batchelor, G. K.: An Introduction to Fluid Dynamics, Cambridge University Press, doi:10.1017/CBO9780511800955, 2000.

Bates, J. R.: Climate stability and sensitivity in some simple conceptual models, Climate Dynamics, 38, 455-473, doi:10.1007/s00382-0100966-0, 2012.

Beare, R. J., Macvean, M. K., Holtslag, A. A. M., Cuxart, J., Esau, I., Golaz, J.-C., Jimenez, M. A., Khairoutdinov, M., Kosovic, B., Lewellen, D., Lund, T. S., Lundquist, J. K., Mccabe, A., Moene, A. F., Noh, Y., Raasch, S., and Sullivan, P.: An intercomparison of large-eddy simulations of the stable boundary layer, Boundary-Layer Meteorol., 118, 247-272, doi:10.1007/s10546-004-2820-6, 2006.

Boer, G. J. and Lambert, S.: The energy cycle in atmospheric models, Climate Dynamics, 30, 371-390, doi:10.1007/s00382-007-0303-4, 2008.

Bony, S., Stevens, B., Frierson, D. M. W., Jakob, C., Kageyama, M., Pincus, R., Shepherd, T. G., Sherwood, S. C., Siebesma, A. P., Sobel, A. H., Watanabe, M., and Webb, M. J.: Clouds, circulation and climate sensitivity, Nature Geoscience, 8, 261-268, doi:10.1038/ngeo2398, 2015.

Bosilovich, M. G., Robertson, F. R., and Chen, J.: Global energy and water budgets in MERRA, J. Climate, 24, 5721-5739, doi:10.1175/2011JCLI4175.1, 2011.

Boville, B. A. and Bretherton, C. S.: Heating and kinetic energy dissipation in the NCAR Community Atmosphere Model, J. Climate, 16, 3877-3887, doi:10.1175/1520-0442(2003)016<3877:HAKEDI>2.0.CO;2, 2003.

Cook, B. I., Anchukaitis, K. J., Touchan, R., Meko, D. M., and Cook, E. R.: Spatiotemporal drought variability in the Mediterranean over the last 900 years, Journal of Geophysical Research: Atmospheres, pp. n/a-n/a, doi:10.1002/2015JD023929, http://dx.doi.org/10.1002/ 2015JD023929, 2015JD023929, 2016.

de Boisséson, E., Balmaseda, M. A., Abdalla, S., Källén, E., and Janssen, P. A. E. M.: How robust is the recent strengthening of the Tropical Pacific trade winds?, Geophys. Res. Lett., 41, 4398-4405, doi:10.1002/2014GL060257, 2014.

Dobrovolski, R. and Rattis, L.: Water collapse in Brazil: the danger of relying on what you neglect, Natureza \& Conservação, 13, 80-83, doi:10.1016/j.ncon.2015.03.006, 2015.

Goody, R.: On the mechanical efficiency of deep, tropical convection, J. Atmos. Sci., 60, 2827-2832, doi:10.1175/15200469(2003)060<2827:OTMEOD>2.0.CO;2, 2003.

Held, I. M. and Hou, A. Y.: Nonlinear axially symmetric circulations in a nearly inviscid atmosphere., J. Atmos. Sci., 37, 515-533, doi:10.1175/1520-0469(1980)037<0515:NASCIA>2.0.CO;2, 1980.

Huang, J. and McElroy, M. B.: A 32-year perspective on the origin of wind energy in a warming climate, Renewable Energy, 77, 482-492, doi:10.1016/j.renene.2014.12.045, 2015.

Johnson, N. C. and Xie, S.-P.: Changes in the sea surface temperature threshold for tropical convection, Nature Geoscience, 3, 842-845, doi:10.1038/ngeo1008, 2010.

Kasahara, A.: Various vertical coordinate systems used for numerical weather prediction, Mon. Wea. Rev., 102, 509-522, doi:10.1175/15200493(1974)102<0509:VVCSUF>2.0.CO;2, 1974. 
Atmos. Chem. Phys. Discuss., doi:10.5194/acp-2016-203, 2016

Atmospheric

Chemistry

Manuscript under review for journal Atmos. Chem. Phys.

Published: 5 April 2016

(c) Author(s) 2016. CC-BY 3.0 License.

Kieu, C.: Revisiting dissipative heating in tropical cyclone maximum potential intensity, Quart. J. Roy. Meteorol. Soc., 141, 2497-2504, doi:10.1002/qj.2534, 2015.

Kim, Y.-H. and Kim, M.-K.: Examination of the global lorenz energy cycle using MERRA and NCEP-reanalysis 2, Climate Dynamics, 40, 1499-1513, doi:10.1007/s00382-012-1358-4, 2013.

Kleidon, A., Renner, M., and Porada, P.: Estimates of the climatological land surface energy and water balance derived from maximum convective power, Hydrol. Earth Syst. Sci., 18, 2201-2218, doi:10.5194/hess-18-2201-2014, 2014.

Kociuba, G. and Power, S. B.: Inability of CMIP5 models to simulate recent strengthening of the Walker circulation: implications for projections, J. Climate, 28, 20-35, doi:10.1175/JCLI-D-13-00752.1, 2015.

Laliberté, F., Zika, J., Mudryk, L., Kushner, P. J., Kjellsson, J., and Döös, K.: Constrained work output of the moist atmospheric heat engine in a warming climate, Science, 347, 540-543, doi:10.1126/science.1257103, 2015.

Lorenz, E. N.: The Nature and Theory of the General Circulation of the Atmosphere, World Meteorological Organization, 1967.

Makarieva, A. M., Gorshkov, V. G., Nefiodov, A. V., Sheil, D., Nobre, A. D., Bunyard, P., and Li, B.-L.: The key physical parameters governing frictional dissipation in a precipitating atmosphere, J. Atmos. Sci., 70, 2916-2929, doi:10.1175/JAS-D-12-0231.1, 2013a.

Makarieva, A. M., Gorshkov, V. G., Sheil, D., Nobre, A. D., and Li, B.-L.: Where do winds come from? A new theory on how water vapor condensation influences atmospheric pressure and dynamics, Atmos. Chem. Phys., 13, 1039-1056, doi:10.5194/acp-13-1039-2013, 2013 b.

Makarieva, A. M., Gorshkov, V. G., and Nefiodov, A. V.: Condensational power of air circulation in the presence of a horizontal temperature gradient, Phys. Lett. A, 378, 294-298, doi:10.1016/j.physleta.2013.11.019, 2014.

Makarieva, A. M., Gorshkov, V. G., and Nefiodov, A. V.: Empirical evidence for the condensational theory of hurricanes, Phys. Lett. A, 379, 2396-2398, doi:10.1016/j.physleta.2015.07.042, 2015a.

Makarieva, A. M., Gorshkov, V. G., Nefiodov, A. V., Sheil, D., Nobre, A. D., and Li, B.-L.: Comments on "The tropospheric land-sea warming contrast as the driver of tropical sea level pressure changes", J. Climate, 28, 4293-4307, doi:10.1175/JCLI-D-14-00592.1, 2015b.

Makarieva, A. M., Gorshkov, V. G., Nefiodov, A. V., Sheil, D., Nobre, A. D., Shearman, P. L., and Li, B.-L.: Heat engines and heat pumps in a hydrostatic atmosphere: How surface pressure and temperature control wind power output and circulation cell size, http://arxiv.org/abs/ 1505.02679, eprint arXiv: 1505.02679 [physics.ao-ph], 2015c.

Marengo, J. A. and Espinoza, J. C.: Extreme seasonal droughts and floods in Amazonia: causes, trends and impacts, Int. J. Climatol., pp. n/a-n/a, doi:10.1002/joc.4420, 2015.

Marvel, K., Kravitz, B., and Caldeira, K.: Geophysical limits to global wind power, Nature Climate Change, 3, 118-121, doi:10.1038/nclimate1683, 2013.

Ohmura, A. and Raschke, E.: Chapter 10. Energy Budget at the Earth's Surface, in: Observed Global Climate, edited by Hantel, M., vol. 6 of Landolt-Börnstein - Group V Geophysics, pp. 1-30, Springer, doi:10.1007/b75667, 2005.

Pauluis, O.: Water vapor and mechanical work: A Comparison of Carnot and steam cycles, J. Atmos. Sci., 68, 91-102, doi:10.1175/2010JAS3530.1, 2011.

Pauluis, O. and Dias, J.: Satellite estimates of precipitation-induced dissipation in the atmosphere, Science, 335, 953-956, doi:10.1126/science.1215869, 2012.

Pauluis, O., Balaji, V., and Held, I. M.: Frictional dissipation in a precipitating atmosphere, J. Atmos. Sci., 57, 989-994, doi:10.1175/15200469(2000)057<0989:FDIAPA>2.0.CO;2, 2000. 
Atmos. Chem. Phys. Discuss., doi:10.5194/acp-2016-203, 2016

Manuscript under review for journal Atmos. Chem. Phys.

Published: 5 April 2016

(c) Author(s) 2016. CC-BY 3.0 License.

(c) (i)
Atmospheric

Chemistry

and Physics

Discussions

Pelkowski, J.: Of entropy production by radiative processes in a conceptual climate model, Meteorologische Zeitschrift, 21, 439-457, doi:10.1127/0941-2948/2012/0401, 2012.

Rienecker, M. M., Suarez, M. J., Gelaro, R., Todling, R., Bacmeister, J., Liu, E., Bosilovich, M. G., Schubert, S. D., Takacs, L., Kim, G.-K., Bloom, S., Chen, J., Collins, D., Conaty, A., da Silva, A., Gu, W., Joiner, J., Koster, R. D., Lucchesi, R., Molod, A., Owens, T., Pawson, S., Pegion, P., Redder, C. R., Reichle, R., Robertson, F. R., Ruddick, A. G., Sienkiewicz, M., and Woollen, J.: MERRA: NASA’s modern-era retrospective analysis for research and applications, J. Climate, 24, 3624-3648, doi:10.1175/JCLI-D-11-00015.1, 2011.

Robertson, F. R., Bosilovich, M. G., Chen, J., and Miller, T. L.: The effect of satellite observing system changes on MERRA water and energy fluxes, J. Climate, 24, 5197-5217, doi:10.1175/2011JCLI4227.1, 2011.

Sabin, T. P., Babu, C. A., and Joseph, P. V.: SST-convection relation over tropical oceans, Int. J. Climatol., 33, 1424-1435, doi:10.1002/joc.3522, 2013.

Schubert, G. and Mitchell, J. L.: Planetary Atmospheres as Heat Engines, in: Comparative Climatology of Terrestrial Planets, edited by Mackwell, S. J., Simon-Miller, A. A., Harder, J. W., and Bullock, M. A., pp. 181-191, University of Arizona Press, Tucson, doi:10.2458/azu_uapress_9780816530595-ch008,2013.

Shepherd, T. G.: Atmospheric circulation as a source of uncertainty in climate change projections, Nature Geoscience, 7, 703-708, doi:10.1038/ngeo2253, 2014.

Tailleux, R.: Observational and energetics constraints on the non-conservation of potential/Conservative Temperature and implications for ocean modelling, Ocean Modelling, 88, 26-37, doi:10.1016/j.ocemod.2015.02.001, 2015.

Wu, W. and Liu, Y.: Radiation entropy flux and entropy production of the Earth system, Rev. Geophys., 48, RG2003, doi:10.1029/2008RG000275, 2010. 